\title{
A Dynamic Model of the Limit Order Book
}

\author{
Ioanid Rosu*
}

December 31, $2003^{\dagger}$

\begin{abstract}
I propose a continuous-time model of price formation in a market where trading is conducted according to a limit-order book. Strategic liquidity traders arrive randomly in the market and dynamically choose between limit and market orders, trading off execution price with waiting costs. I prove the existence of a Markov equilibrium in which the bid and ask prices depend only on the numbers of buy and sell orders in the book, and which can be characterized in closed-form in several cases of interest. My model generates empirically verified implications for the shape of the limit-order book and the dynamics of prices and trades. In particular, I show that buy and sell orders can cluster away from the bid-ask spread, thus generating a hump-shaped limit-order book. Also, following a market buy order, both the ask and bid prices increase, with the ask increasing more than the bid-hence the spread widens.
\end{abstract}

JEL Classification: C7, D4, G1.

Keywords: Liquidity, price impact, waiting costs, limit order book, order-driven markets, game of attrition.

${ }^{*}$ MIT, Sloan School of Management. E-mail: ioanid@mit.edu.

${ }^{\dagger}$ I am grateful to Drew Fudenberg, Xavier Gabaix, Sergei Izmalkov, Leonid Kogan, Jon Lewellen, Andrew Lo, David McAdams, Stew Myers, Jun Pan, Anna Pavlova, Dimitri Vayanos, Jiang Wang and seminar participants at the Finance and Theory lunches and Batterymarch Finance seminar at MIT for helpful discussions and comments. All remaining errors and omissions are mine. 


\section{Introduction}

In most classical models of market microstructure ${ }^{1}$ the market maker plays a central role. The main function of the market maker is to provide liquidity (immediacy) for those who wish to trade, by setting bid and ask quotes. However, there exist many markets, called order-driven, or pure limit order markets, in which there are no market makers (e.g., ECNs, Euronext, Hong Kong, Tokyo, Toronto). In these markets every investor can supply liquidity by placing limit orders in a limit order book. ${ }^{2}$ With the recent trend towards larger and more automated exchanges, order-driven markets have become increasingly important. ${ }^{3}$ There are also hybrid exchanges (e.g., NYSE, Nasdaq, London), where market makers exist but have to compete with other traders, who supply liquidity by limit orders. In these markets, the number of transactions which involve a market maker is usually small. ${ }^{4}$

The study of liquidity provision when market makers do not exist or only have a limited role is therefore very important in understanding modern financial markets. ${ }^{5}$ However, it is quite a complicated problem. ${ }^{6}$ To solve it, one would need to know exactly how market prices arise from the interaction of a large number of anonymous traders, who arrive in the market at random times, can choose whether to trade immediately or to wait, and who can behave strategically by changing their orders at any time.

In this paper I propose a model of an order-driven market which reflects all the features mentioned above. The model is tractable and produces sharp implications about (i) the shape of the limit order book at any point in time, and (ii) the evolution in time of the book, and in particular of the bid and ask prices. The model is in line with known empirical facts, such

\footnotetext{
${ }^{1}$ See the survey book by O'Hara (1995).

${ }^{2}$ Limit orders are price-contingent orders to buy (sell) if the price falls below (rises above) a prespecified price. The limit order book is the collection of all price-contingent orders which have not yet been executed.

${ }^{3}$ Nowadays, about half of the world's stock exchanges are organized as order-driven markets, with no designated market makers, while only a few exchanges completely rely on dealer quotes (see Jain (2002)).

${ }^{4}$ For example, on the NYSE the specialists' participation is about 13\%, and even less in the case of larger stocks (see Hasbrouck and Sofianos (1993)).

${ }^{5}$ One can argue that market making behavior is important and may arise endogenously even in order-driven markets. However, I know of no data to support this. Anecdotically, there seem to be many traders who submit limit orders (thus providing liquidity), but who do not maintain a continuous presence in the market. Consider the case of a value mutual fund who wishes to acquire a stock based on some analyst reports. If it is willing to wait in the hope of a better execution price in the near future, then it can do so by placing a limit buy order. After the order is executed, the fund might not want to trade again in that stock any time soon. And even if it does, it is not so hard to accept that the next decision to trade in that particular stock can be thought of as being made by a different fund.

${ }^{6}$ There has been significant progress in recent years: see for example the models of Foucault et al. (2003), Goettler et al. (2003), Parlour (1998), and also the discussion in Harris (1998). The main difficulty is not in formulating the problem, but in obtaining a tractable model.
} 
as the hump shape of the limit order book. It can also explain why following a market buy order both the bid and the ask increase, not only the ask.

I consider a continuous-time, infinite-horizon economy where there is only one asset with no dividends. Buyers and sellers arrive to the market randomly. They either buy or sell one unit of the asset, after which they exit the model. I assume that all traders are liquidity traders, in the sense that their impulse to trade is exogenous to the model. However, they are discretionary, because they have a choice over when to trade, and whether to place a market or limit order. After a limit order is placed, it can be canceled and changed at will. The execution of limit orders is subject to the usual price priority rule, and when prices are equal to the first-in-first-out (FIFO) rule. All agents incur waiting costs, i.e., a loss of utility from waiting. Depending on whether they have low or high waiting costs, traders are patient or impatient. All information is common knowledge, including the limit order book (which is the collection of outstanding limit orders).

In equilibrium it turns out, not surprisingly, that patient agents submit in general limit orders, while impatient agents submit market orders. The new limit orders are always placed inside the bid-ask spread ${ }^{7}$, until the spread reaches a minimum level. When this happens let us call the book "full." At that point, patient agents either place a market order or start submitting quick (fleeting) limit orders which in effect behave like market orders. This comes theoretically as a result of a game of attrition amongst the buyers or the sellers.

In order to obtain intuition about the limit order book, I study in more detail a particular case of the model, where one considers just one side of the book, e.g., the sell side: with only patient sellers and impatient buyers. Then the solution can be expressed in closed-form.

Also, to discuss price impact and determine the shape of the limit order-book, I allow for multi-unit market orders, even with very small probabilities. This assumption fixes the levels on which agents place their limit orders, which will then be different for each agent. ${ }^{8}$ I show that if the such orders arrive with probabilities which do not decrease too fast (or rather that the agents do not believe so), then the book exhibits a hump shape, i.e., the limit orders will cluster away from the bid and the ask (cf. Biais et al. (1995), and Bouchaud et al. (2002)).

\footnotetext{
${ }^{7}$ This is not unrealistic, the majority of limit orders are spread-improving: see Biais et al. (1995). One can modify this model, so that the population of patient agents is heterogeneous. Then more patient agents may then place orders away from the market. The model is however more difficult to solve.

${ }^{8}$ The fact that agents limit trade at different levels comes from the fact that the FIFO rule breaks the symmetry of the payoffs, which forces orders to have different times to execution.
} 
The case when there are no impatient agents can also be solved in closed-form. The resulting equilibrium is quite interesting: buyers and sellers cannot coexist in the limit order book. There are either a lot of sellers in the book and the buyers place market orders, or vice versa. This shows that the existence of impatient agents is important in order for a limit order book to function properly.

I also derive the equilibrium processes that bid and ask prices follow, and the expected time-to-execution for limit orders. ${ }^{9}$ The point where the limit order book is full coincides with the time when the bid-ask spread is minimum (an interesting fact since the tick size is zero). One may call this minimum spread the competitive bid-ask spread. Then, as in Foucault et al. (2003), I define resiliency as the speed with which the bid-ask spread reverts to its competitive level. I then recover their results: the resiliency of the limit order book increases with the proportion of patient traders, but decreases with order arrival rate, etc.

An interesting empirical implication is that after a market sell order both the bid and ask prices decrease, with the bid decreasing more than the ask. ${ }^{10}$ As a result, the spread itself widens. The latter fact was obtained by Foucault et al., but since they do not allow for cancellation of limit orders, did not also obtain a decrease in the ask price. The fact that the ask also decreases is documented for example in Biais et al. (1995), who also propose an information explanation. ${ }^{11}$

The limit order book was analyzed in a variety of ways. The information models, which consider market makers interacting with informed agents, are all static: see Glosten (1994), Chakravarty and Holden (1995), Rock (1996), Seppi (1997) and Parlour and Seppi (2001). Moreover, traders are restricted to placing limit orders, so they do not have a choice to submit market orders. Dynamic models, without market makers, are studied by Parlour (1998), Foucault (1999), Foucault, Kadan and Kandel (2003), Goettler, Parlour and Rajan (2003). However, these models are typically not very tractable, and do not allow for strategic cancellation of limit orders.

\footnotetext{
${ }^{9}$ In this model, since agents can switch places in the book, they can increase expected time-to-execution while keeping utility constant (by getting a higher expected execution price). However, if one assumes that traders preserve the relative order in which they arrived in the book, one can define a meaningful notion of time-to-execution.

${ }^{10}$ The bid decreases mechanically, because a limit buy order is cleared from the book. The fact that the ask decreases reflects the sellers' realization that their reservation value (the bid) has decreased, so they also adjust the ask.

${ }^{11}$ This implication can be generated also in a model with price discovery, such as in Glosten and Milgrom (1985). However, I show that even in the absence of information such dynamics may occur.
} 
Although the present paper was developed independently from this literature, it turns out that it is closely related to the work of Foucault, Kadan and Kandel (2003). In their model, waiting costs are also the driving force. An important feature of their model is the existence of discrete prices. This allows them to make a comparison among various tick sizes. However, discrete prices make their model more complicated, so in order to simplify it they have to impose strong assumptions such as: (i) there is no cancellation of limit orders (so agents only make only one decision, when they arrive); (ii) a buyer must always arrive after a seller, and vice versa ; and (iii) new orders have to be improving the existing limit orders by at least a tick. Assumptions (ii) and (iii) make the spread the only state variable, which allows a recursive structure for the solution. As a consequence, their model only focuses on the bid-ask spread, and not on the evolution of the actual bid and ask prices.

An interesting related literature is on liquidity and search costs (e.g., Duffie, Garleanu and Pedersen (2001), Vayanos and Wang (2003)), where buyers and sellers have to search for counter-parties to trade. My contention is that on organized exchanges search costs become one-dimensional and can be better thought of as waiting costs, which leads to the current model. ${ }^{12}$ This paper is also related to the burgeoning field of econophysics (see Farmer et al. (2003) or Gabaix et al. (2003)). See also the literature on double auctions and bid-ask markets, e.g., Wilson (1986).

The paper is organized as follows. Section 2 describes the model. Section 3 solves for the equilibrium in a particular case (with patient sellers and impatient buyers), which describes the the sell side of the book. Section 4 applies the previous results to analyze price impact and the shape of the limit order book. Section 5 describes the equilibrium in the case with both sellers and buyers, patient and impatient, and Section 6 solves for the equilibrium in the case when there are only patient agents. Section 7 addresses some empirical implications, and Section 8 concludes.

\footnotetext{
${ }^{12}$ Of course, one could still argue that search costs are important, especially when stocks are traded on more than one exchange, or when there is more than one specialist. But, more importantly, search costs become significant when dealing with large (block) trades. Since block trades are not dealt with in my model, it would be interesting if one could merge the two frameworks and shed more light on this issue.
} 


\section{The Model}

\subsection{The Market}

I consider a market in an asset which yields no dividends. The buy and sell prices for this asset are determined as the bid and ask prices resulting from trading based on the rules given below. There is a constant range $A>B$ where the prices lie at all times. More specifically, there is an infinite supply when price is $A$, provided by agents outside the model. Similarly, there is an infinite demand for the asset when price is $B$. Prices can take any value in this range, i.e., the tick size is zero.

Trading. The time horizon is infinite, and trading in the asset takes place in continuous time. The only types of trades allowed are market orders and limit orders. The limit orders are subject to the usual price priority rule; when prices are equal, the first-in-first-out (FIFO) rule is applied. If several market orders are submitted at the same time, only one of them is executed, at random, while the other orders are canceled. ${ }^{13}$ Limit orders can be canceled for no cost at any time. There is also no delay in trading, both types of orders being posted or executed instantaneously. Trading is based on a publicly observable limit order book, ${ }^{14}$ which collects all the limit orders that have not been canceled or executed.

Agents. The market is populated by traders who arrive randomly to the market, and choose strategically between market and limit orders. They are liquidity traders, in the sense that they want to trade the asset for reasons exogenous to the model. The traders are either buyers or sellers; their type is fixed from the beginning and cannot change. Buyers and sellers trade at most one unit, after which exit the model forever.

Traders are risk-neutral, so their instantaneous utility function (felicity) is linear with price. ${ }^{15}$ By convention, felicity is equal to price for sellers, and minus the price for sellers. Traders discount the future in a way proportional to the expected waiting time. Thus, if $\tau$ is

\footnotetext{
${ }^{13}$ To justify this assumption, it is best to think of a market buy (sell) order as a limit order with limit price equal to the ask (bid). Then if several market orders are submitted at the same time, one of them is randomly executed, while the others remains as limit orders, which can be freely canceled.

${ }^{14}$ Typically the limit order book is not fully observed, except to specialists. However, in order-driven exchanges such as Euronext or Island the first few limit orders close to the bid and ask are public. In other exchanges such as Taiwan Stock Exchange, this information can be purchased.

${ }^{15}$ This model also works with exponential time discounting, but the resulting formulas are more complicated.
} 
the random execution time and $P_{\tau}$ is the price obtained at $\tau$, the utility of a seller is

$$
f_{t}=\mathrm{E}_{t}\left\{P_{\tau}-r(\tau-t)\right\}
$$

Similarly, the utility of a buyer is $-g_{t}=\mathrm{E}_{t}\left\{-P_{\tau}-r(\tau-t)\right\}$, where I introduce the notation

$$
g_{t}=\mathrm{E}_{t}\left\{P_{\tau}+r(\tau-t)\right\}
$$

I call $f_{t}$ the value function of the seller at $t$, and $g_{t}$ the value function of the buyer, although in fact $g_{t}$ equals minus the utility of a buyer.

The discount coefficient $r$ is constant, and can take two values: if it is low, the corresponding traders are called patient, otherwise they are impatient. Agents' types are determined from the beginning and cannot change.

For simplicity, I assume that the impatient agents always submit market orders. This is not necessary to make the model work, but it simplifies the presentation. In the Appendix, I discuss conditions for the coefficient $r$ such that in equilibrium impatient agents always submit market orders. Assuming this, from now on $r$ denotes only the time discount coefficient of the patient agents.

Arrivals. The four types of traders (patient buyers, patient sellers, impatient buyers, and impatient sellers) arrive to the market according to independent Poisson processes with constant arrival intensity rates

$$
\lambda_{P B}, \lambda_{P S}, \lambda_{I B}, \lambda_{I S}
$$

By definition, Poisson arrival with intensity $\lambda$ implies that the number of arrivals in any interval of length $T$ has a Poisson distribution with parameter $\lambda T$. The inter-arrival times of a Poisson process are distributed as an exponential variable with the same parameter $\lambda$. The mean time until the next arrival is then $1 / \lambda$. The interval until the next arrival is called a period.

Strategies. Because of Poisson arrivals, the game must be set in continuous time. Since there is no universally accepted standard of continuous time game theory, I define in Appendix B the game theoretic setup that seems the most appropriate to our case: stochastic multi-stage game with observed actions, by extending a framework created by Bergin and 
MacLeod (1993). All information, together with agents' strategies and beliefs are common knowledge.

One special feature of this model that has to be addressed comes from market orders. Suppose at time $t$ an agent submits a market order. Then the agent exits the model, and the next stage of the game will be played with fewer players. But at which time will this next game be played? No $t+\varepsilon>t$ is satisfactory, because it would imply waiting for a positive time, during which agents lose utility. The best solution, as in auction theory, is to "stop the clock." Then the next game is also played at time $t$, and the clock is restarted only when in the stage game no agent submits a market order. Allowing for clock stopping in continuous time game theory requires some care, and it is done in Appendix B.

An important benefit of setting the game in continuous time is that agents can respond immediately. More precisely, one can use strategies that specify: "Keep the limit order at $a_{1}$ as long as the other agent stays at $a_{2}$ or below. If at some time $t$ the other agent places an order above $a_{2}$, then immediately after $t$ undercut at $a_{2}$." In the rest of the paper, I use this type of strategies freely.

The notions of equilibrium used are sub-game perfect equilibrium, and Markov perfect equilibrium (see Fudenberg and Tirole, ch. 13). One other notion that is important in this framework is that of competitive equilibrium, which is a sub-game perfect equilibrium such that at each time all buyers have the same value function (and similarly for sellers). These are discussed in more detail in Appendix B.

\subsection{Discussion.}

I now discuss some of the features of this model. One strong assumption is that prices lie within a range $[B, A]$, and that $A$ and $B$ are known by everybody with certainty. Clearly, a more realistic assumption would be to make $A$ and $B$ random, or even stochastic, perhaps as prices coming from valuations of informed traders. In fact, one can think of $A$ and $B$ as summarizing information about the asset, while within this range prices fluctuate due to the exogenously specified order flow (of course, one should not artificially separate order flow from information). This interpretation would imply that empirical implications of this model would be more believable when obtained in high frequencies, when less information is likely to arrive.

One should be clear that this is not an asymmetric information model. As pointed out 
by Foucault et al. (2003), in this kind of models, frictions such as the bid-ask spread are completely determined by (i) the waiting costs of agents, and (ii) strategic rent-seeking by patient traders. But this is not so unrealistic. Huang and Stoll (1997) for example estimate that on average approximately $90 \%$ of the bid-ask spread is due to non-informational frictions ("order-processing costs").

Another strong assumption is having independent Poisson arrivals. From a qualitative standpoint, more important than the actual Poisson distribution is the independence of the increments of the arrival process. This implies that at each point during the period between two successive arrivals, the agents face the same market conditions, which allows for a relatively simple description of the equilibrium.

\section{Equilibrium: One Side of the Book}

In this section, I analyze the sell-side of the limit order book, by assuming only two types of traders: patient sellers and impatient buyers. With the notation given above, $\lambda_{P B}=\lambda_{I S}=0$. (By symmetry, one can derive similar results for the buy-side.) This case proves to be quite tractable. Moreover, it is also useful for understanding the general case, which can be thought as merging two one-sided models.

\section{Intuition}

Here is some intuition for the equilibrium. Imagine the limit order book is empty, and a patient seller (labeled "1") arrives first to this market. Then, until some other agent arrives, trader 1 optimally behaves like a monopolist, and submits a limit sell order at $a_{1}=A .{ }^{16}$ Suppose now a second patient seller (labeled "2") arrives. Now both sellers compete for the possibility of a market order from incoming impatient buyers. If trader 1 could not cancel his limit order at $A$, then trader 2 would surely place her limit order at $a_{2}=A-\delta$, for some very small $\delta$. But trader 1 can change his limit order, so a price war would likely follow. In order for both traders to be satisfied, trader 2 needs to place her limit order at a level $a_{2}<A$ low enough so that trader 1 would be indifferent between keeping his limit order at $a_{1}$ (thus having a chance to become a monopolist again after trader 2's order gets cleared), and switching places with trader 2. Now, being indifferent in this case exactly means that both

\footnotetext{
${ }^{16}$ I have assumed implicitly that if the only limit sell orders in the book are at $A$, a market order first clears the orders in the book, and only after relies on the infinite supply at $A$.
} 


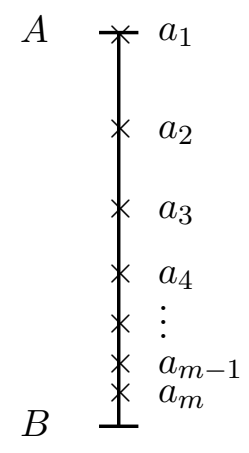

Figure 1: The limit order book with $m$ sellers.

traders get the same expected utility from their strategies. Recall that when traders derive the same expected utility (value) from their equilibrium strategies the equilibrium is called competitive. ${ }^{17}$ Of course, the values $a_{1}$ and $a_{2}$ are determined in equilibrium, and depend on what other agents will do: imagine that instead of an impatient buyer who places a market order at $a_{2}$, a patient seller comes, who will place a limit order at $a_{3}$, etc.

To summarize the above discussion, when there are $m$ sellers in the book, in equilibrium it must be that some trader has a limit order at the ask $a_{m}$. As long as the orders of others are above $a_{m}$, their exact levels do not matter (this is because the incoming market orders are one-unit). However, if the trader at the ask tries to place the order higher, then some other agent immediately will jump at $a_{m}$.

\subsection{Equal Arrival Rates}

I assume a dynamic market clearing condition, namely that the arrival rates of patient sellers and impatient buyers are equal:

$$
\lambda=\lambda_{P S}=\lambda_{I B}>0
$$

Start with a competitive stationary Markov equilibrium. The idea is to find necessary conditions for such an equilibrium to exist, and then to prove that the conditions are also sufficient. As mentioned in the previous section, the Markov equilibrium is defined with respect to the

\footnotetext{
${ }^{17}$ One can imagine a different scenario, when all patient sellers queue their limit orders at $A$ until the expected utility of the last trader equals the reservation value $B$. How can such a non-competitive equilibrium be sustained? By Nash threats: Trader 1 can threaten with competitive behavior if trader 2 does not queue behind him at $A$. Trader 2 is better off by complying as long as she expects trader 3 to do the same and queue behind her. Although these other, non-competitive, equilibria are interesting in their own right, I focus on competitive equilibria since they are the more likely outcome of large, anonymous order-driven markets.
} 
state variable given by the number of sellers $m$ in the limit order book. Since in a competitive equilibrium all agents have the same utility, denote by $f_{m}$ the utility of an agent in state $m$.

I enumerate a few results about the equilibrium that will be proved in Appendix A. First, the number of states must be finite. Denote by $M$ the largest state. As $m$ increases, each seller is strictly worse off. In state $M$ it must be true that $f_{M}=B$, otherwise another patient seller would be tempted to join in. In this state the bottom seller has a mixed strategy: at the first arrival in an independent Poisson process with intensity $\mu$, the seller will place a market order and exit. ${ }^{18}$ Observe that from a state $m=1, \ldots, M-1$, the system can go to one of two states:

- $m-1$, if an impatient buyer arrives - after random time $T_{1}$;

- $m+1$, if a patient seller arrives - after random time $T_{2}$.

Inter-arrival times of Poisson processes are exponentially distributed, so the arrival of the first of the two states happens at $\min \left(T_{1}, T_{2}\right)$, which is exponential with intensity $2 \lambda$. Then each event happens with probability $1 / 2$. One obtains the formula $f_{m}=\frac{1}{2}\left(f_{m-1}+f_{m+1}\right)-r \cdot \frac{1}{2 \lambda}$. Denote by

$$
\varepsilon=\frac{r}{\lambda}
$$

The formula becomes

$$
2 f_{m}+\varepsilon=f_{m-1}+f_{m+1}
$$

From the terminal state $M$, the system can go to

- $M-1$, if an impatient buyer arrives - after random time $T_{1}$;

- $M$, if a patient seller arrives - after random time $T_{2}$;

- $M-1$, if a current seller places a market order and exits - after random time $T_{3}$.

One can ignore the arrival of a new patient seller, since it does not affect the state (the seller places a market order at $B$ and exits). Then one gets the formula $f_{M}=f_{M-1}-r \cdot \frac{1}{\lambda+\mu}$. Define

$$
u=\frac{\lambda}{\lambda+\mu} \in(0,1]
$$

\footnotetext{
${ }^{18}$ There is another possibility for a mixed strategy, but it leads to the same formulas. See Proposition 19.
} 
The formula becomes

$$
f_{M}+u \varepsilon=f_{M-1}
$$

Define also (this will be justified later):

$$
f_{0}=A
$$

All these equations can be put together in the following definition.

Definition 1. Let $u \in(0,1]$ and $M>0$ an integer. A sequence $f_{m}, m=1, \ldots, M$, is called $a(u, M)$-chain if

$$
\left\{\begin{array}{l}
f_{0}=A \\
2 f_{m}+\varepsilon=f_{m+1}+f_{m-1}, \quad m=1, \ldots, M-1 \\
f_{M}+u \varepsilon=f_{M-1} .
\end{array}\right.
$$

The $(u, M)$-chain $f_{m}$ is called maximal if $f_{M}=B$.

An important intuition for a $(u, M)$-chain is that it $f_{m}$ decreases for $m<M$, after which it starts increasing.

Proposition 1. Suppose $f_{m}$ is extended above $M$ via the middle equation of (1). Then if $i<M$ is an integer, not necessarily positive, the following recursive relation holds:

$$
f_{M-i-1}=f_{M-i}+\varepsilon(i+u)
$$

In particular, a $(u, M)$-chain $f_{m}$ is strictly decreasing in $m$ if $m<M$ and strictly increasing if $m>M$.

What was done so far was to show that the value functions for any competitive stationary Markov equilibrium form a maximal $(u, M)$-chain. The next result shows that given $A, B$ and $\varepsilon$, such a chain is unique and gives explicit formulas to calculate it.

Proposition 2. Given $A>B$ and $\varepsilon>0$, there exists a unique maximal (u, $M)$-chain. Any sequence $f_{m}$ that forms a maximal $(u, M)$-chain satisfies the formula

$$
f_{m}=A-b m+\frac{\varepsilon}{2} m^{2}
$$


where

$$
b=\varepsilon \sqrt{\left(\frac{1}{2}-u\right)^{2}+\frac{2(A-B)}{\varepsilon}}=\varepsilon\left(M-\frac{1}{2}+u\right) .
$$

The integer $M>0$ and real number $u \in(0,1]$ are the unique ones for which

$$
M=\frac{1}{2}-u+\sqrt{\left(\frac{1}{2}-u\right)^{2}+\frac{2(A-B)}{\varepsilon}}
$$

is an integer.

Proof. See Appendix A.

I now state the main result of this Section.

Theorem 3. Given $A, B, r, \lambda$, there exists a unique competitive stationary Markov equilibrium of the game. Let $\varepsilon=\frac{r}{\lambda}$, and $M, u, f_{m}$ as in Proposition 2. Then in equilibrium there are at most $M$ limit orders in the book, and the ask price in state $m=1, \ldots, M$ is given by

$$
\begin{aligned}
& a_{m}=f_{m-1}, \quad \text { if } m<M ; \\
& a_{M}=B+\varepsilon .
\end{aligned}
$$

The value function in state $m$ is given by $f_{m}$. The strategy of each agent in state $m$ is the following:

- If $m=1$, then place a limit order at $a_{1}=A$.

- If $m=2, \ldots, M-1$, place a limit order at any level above $a_{m}$, as long as someone stays at $a_{m}$ (or below). If not, then place an order at $a_{m}$.

- If $m=M$, the strategy is the same as for $m=2, \ldots, M-1$, except for the bottom seller at $a_{M}$, who exits (by placing a market order at B) after the first arrival in a Poisson process with intensity $\mu=\frac{\lambda}{u}-\lambda$.

- If $m>M$, then immediately place a market order at $B$.

Proof. See Appendix A and the discussion below.

Notice that there is some ambiguity in the way strategies are formulated. They can support several equilibria, all of them payoff-equivalent. For example, in one equilibrium, in state $m$ 
each agent places an order at $a_{m}$, regardless of what the others are doing. Another equilibrium is the one in which no agent changes the order until execution. Then in state $m$, the limit order book has limit orders at $a_{1}, \ldots, a_{m}$. This is the equilibrium suggested at the beginning of this section.

This ambiguity arises from the fact that market orders are for only one unit, so in each state only the limit order at the ask matters. In fact, there is ambiguity also in the identity of the players. As mentioned above, it does not matter who has the order at the ask as long as there is one. That divides the agents essentially into two types:

- The Bottom agent, who has the lowest offer in the book, placed at $a_{m}$.

- The Top $m-1$ agents, who are placed above (or at $a_{m}$ after the Bottom agent, due to the FIFO rule).

Here is the state diagram for the Bottom seller, where $E$ represents exit for this agent (when an impatient seller comes and clears the order at the ask):

(B)

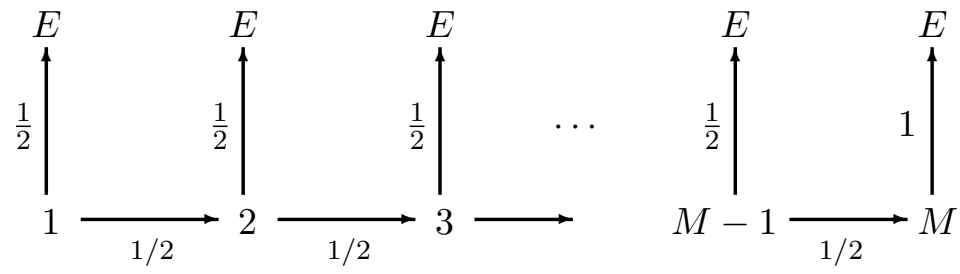

And here is the state diagram for the Top sellers:

(T)

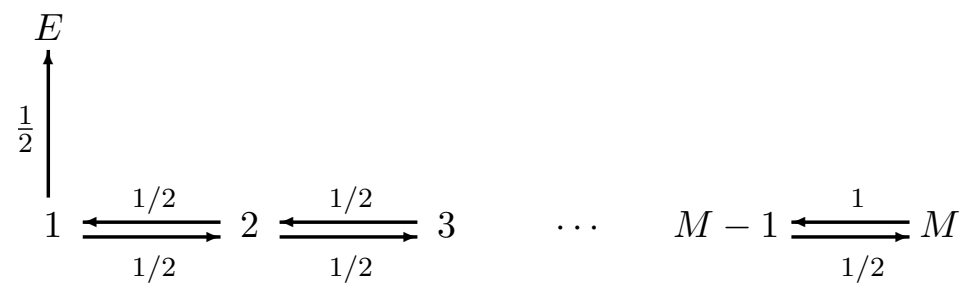

(Notice that in state 1 there is no distinction between the Top and Bottom agents.) One 
obtains then the equations

$$
\begin{aligned}
& \text { (B) } \quad 2 f_{m}+\varepsilon=a_{m}+f_{m+1}, \quad \text { if } m=1, \ldots, M-1 \\
& \text { (T) } \quad 2 f_{m}+\varepsilon=f_{m-1}+f_{m+1}, \quad \text { if } m=2, \ldots, M-1 .
\end{aligned}
$$

Since the equilibrium is competitive, by definition in state $m$ the value functions are all equal, so it follows that $a_{m}=f_{m-1}$ for all $m=2, \ldots, M-1$. Since in state 1 the seller is a monopolist, $a_{1}=A$. This justifies the definition: $f_{0}=A$.

Now it is clear why the strategies defined by Theorem 3 give a sub-game perfect equilibrium: In each state $m$ no agent wants to deviate. The bottom trader will not go lower than $a_{m}$, because then he will lose in expectation. If he tries to go above $a_{m}$, then some top agent will immediately become the bottom one.

One can also calculate

$$
\begin{aligned}
a_{M} & =B+\varepsilon, \\
a_{M-1} & =B+\varepsilon+2 u \varepsilon, \\
a_{M-2} & =B+3 \varepsilon+3 u \varepsilon, \\
a_{M-3} & =B+6 \varepsilon+4 u \varepsilon, \\
& \vdots \\
a_{1} & =A .
\end{aligned}
$$

It is interesting to see how $f_{m}$ depends on $m$ and $B$ :

$$
\begin{aligned}
\frac{\partial f_{m}}{\partial m} & =-\varepsilon\left(\left(M-\frac{1}{2}+u\right)-m\right) \\
\frac{\partial f_{m}}{\partial B} & =\frac{m}{M-\frac{1}{2}+u} .
\end{aligned}
$$

(Notice that $\frac{\partial f_{m}}{\partial B}<1$ when $m<M$.) These formulas are important to get intuition about the equilibrium limit order book with both sellers and buyers (then in some sense $B$ represents the bid price). Here is a heuristic argument: suppose the system is in an equilibrium with $m$ 
sellers and $n+1$ buyers. Then the bid price $B=g_{m, n}$. One calculates

$$
\frac{\partial f_{m, n}}{\partial n}=\frac{\partial f_{m, n}}{\partial B} \cdot \frac{\partial g_{m, n}}{\partial n}<\frac{\partial g_{m, n}}{\partial n}
$$

This implies that when a new buyer arrives, the sellers are better off by less than the buyers are worse off (recall that $g_{m, n}$ is minus the buyers' utility). Another, more rigorous argument, will be given in the section that solves for the equilibrium in the general case.

\subsection{Different Arrival Rates}

In this section, I assume that the patient sellers arrive faster than the impatient buyers:

$$
\lambda_{1}=\lambda_{P S}>\lambda_{2}=\lambda_{I B}
$$

As in the case of equal arrival rates, one starts with a competitive stationary Markov equilibrium, and looks for for necessary conditions. Denote by $f_{m}$ is the value function of a seller in the state where there are $m$ sellers in the book. From the results proved in Appendix A, the number of states is finite, so there exists a largest state $M$. Moreover, $f_{M}=B$. From the state $m=1, \ldots, M-1$ the the system can go to one of two states:

- $m+1$, if a patient seller arrives - after random time $T_{1} \sim \exp \left(\lambda_{1}\right)$;

- $m-1$, if an impatient buyer arrives - after random time $T_{2} \sim \exp \left(\lambda_{2}\right)$.

The arrival of the first of these two states happens at $\min \left(T_{1}, T_{2}\right) \sim \exp \left(\lambda_{1}+\lambda_{2}\right)$. Then the first event happens with probability

$$
\omega=\frac{\lambda_{1}}{\lambda_{1}+\lambda_{2}}>\frac{1}{2}
$$

and the second with probability $1-\omega$. Define

$$
\varepsilon=\frac{r}{\lambda_{1}+\lambda_{2}} .
$$

One then gets the equation

$$
f_{m}+\varepsilon=\omega f_{m+1}+(1-\omega) f_{m-1} .
$$


The characteristic equation $\omega x^{2}-x+(1-\omega)=0$ has two roots: $x_{1}=1$ and $x_{2}=\alpha$, where

$$
\alpha=\frac{1-\omega}{\omega}<1
$$

Imposing the condition $f_{0}=A$ as in the equal arrival case, the general solution is

$$
f_{m}=A-C\left(1-\alpha^{m}\right)+\varepsilon \beta m, \quad \text { with } C \text { arbitrary, }
$$

where

$$
\beta=\frac{1}{2 \omega-1}
$$

The constant $C$ is determined by looking at the final state $M$. In this state, suppose the bottom agent has the mixed strategy to place a market order at the first arrival in a Poisson process with intensity $\mu$. Arrival of patient seller does not matter, so one gets the equation $\left(\lambda_{2}+\mu\right) f_{M}+\varepsilon=\left(\lambda_{2}+\mu\right) f_{M-1}$. Define

$$
s=\frac{\mu}{\lambda_{1}+\lambda_{2}}, \quad u=\frac{1}{1-\omega+s} .
$$

Then one gets

$$
f_{M}+u \varepsilon=f_{M-1}
$$

As in the case of equal arrival rates, one can define a $(u, M)$-chain.

Definition 2. Let $u \in\left(0, \frac{1}{1-\omega}\right]$ and $M>0$ an integer. A sequence $f_{m}, m=1, \ldots, M$, is called $a(u, M)$-chain if

$$
\left\{\begin{array}{l}
f_{0}=A \\
f_{m}+\varepsilon=\omega f_{m+1}+(1-\omega) f_{m-1}, \quad m=1, \ldots, M-1 \\
f_{M}+u \varepsilon=f_{M-1} .
\end{array}\right.
$$

The $(u, M)$-chain $f_{m}$ is called maximal if $f_{M}=B$.

As in the previous Section, a $(u, M)$-chain decreases for $m<M$ and increases for $m>M$.

Proposition 4. Suppose $f_{m}$ is extended above $M$ via the middle equation of (11). Then if 
$i<M$ is an integer, not necessarily positive, the following recursive relation holds:

$$
f_{M-i-1}=f_{M-i}+\varepsilon\left((\beta+u)\left(\alpha^{-i}-1\right)+u\right)
$$

In particular, a $(u, M)$-chain $f_{m}$ is strictly decreasing in $m$ if $m<M$ and strictly increasing if $m>M$.

One can also prove the following result.

Proposition 5. Given $A>B, \omega>\frac{1}{2}$ and $\varepsilon>0$, there exists a unique maximal $(u, M)$-chain. Any sequence $f_{m}$ that forms a maximal $(u, M)$-chain satisfies the formula

$$
f_{m}=A-C_{M}\left(1-\alpha^{m}\right)+\varepsilon \beta m,
$$

where

$$
C_{M}=\frac{\varepsilon(\beta+u)}{\alpha^{M-1}-\alpha^{M}}
$$

The integer $M>0$ and real number $u \in\left(0, \frac{1}{1-\omega}\right]$ are the unique ones for which

$$
A-B+\varepsilon \beta M=\frac{\varepsilon(\beta+u)}{\alpha^{M-1}-\alpha^{M}}\left(1-\alpha^{M}\right) .
$$

An interesting feature of these formulas when $\omega>\frac{1}{2}$ is that one can calculate the limit of equation (12) when $\varepsilon \rightarrow 0$. To see why, notice that $f_{M}=B$, so $C_{M}\left(1-\alpha^{M}\right)=A-B+\varepsilon \beta M$. The number $\alpha^{M}$ is of the order of $\varepsilon$, so $M$ is of the order of $\log \frac{1}{\varepsilon}$. This implies that $C_{M}=A-B$ modulo terms of order $\varepsilon \log \frac{1}{\varepsilon}$, which is of smaller order for example than $\varepsilon^{1 / 2}$. In the end, one gets the following formula

$$
f_{m} \approx B+(A-B) \alpha^{m} \quad \text { if } \varepsilon \approx 0 .
$$

I now come back to the description of the equilibrium.

Theorem 6. Given $A, B, r, \lambda_{1}>\lambda_{2}$, there exists a unique competitive stationary Markov equilibrium of the game. Let $\varepsilon, \alpha, \beta$ as above, and $M, u, f_{m}$ as in Proposition 5. Then in equilibrium there are at most $M$ limit orders in the book, and the ask price in state $m=$ 
$1, \ldots, M$ is given by

$$
\begin{aligned}
& a_{m}=f_{m-1}, \quad \text { if } m<M ; \\
& a_{M}=B+(1-\omega) \varepsilon .
\end{aligned}
$$

The value function in state $m$ is given by $f_{m}$. The strategies of agents are the same as those in Theorem 3.

\subsection{Multi-Unit Market Orders}

When agents have one-unit demands, the only important limit order in equilibrium is the one at the ask, and the others limit orders can be at any level above the ask. However, assume that multi-unit market orders may arise even with very small probability. To be more precise, let $k$ be the maximum number of units that an order can have, with positive probability. Then in equilibrium I show that the last $k$ levels in the order book are fixed. Assume that patient sellers still arrive with only one unit to sell. Define

$$
\left\{\begin{array}{l}
\lambda=\text { arrival rate of patient sellers; } \\
\lambda_{i}=\text { arrival rate of } i \text {-unit impatient sellers, } i=1, \ldots, k
\end{array}\right.
$$

Assume that

$$
\lambda_{i}>0 \text { for all } i=1, \ldots, k \text {. }
$$

Moreover, as in the previous section, one wants the sellers to arrive faster than the units demanded by the buyers. This is equivalent to

$$
\lambda>\sum_{i=1}^{k} i \lambda_{i}
$$

As before, the number of states is finite, so there exists a largest state $M$. Moreover, $f_{M}=B$. From the state $m=1, \ldots, M-1$ the the system can go to one of the following states:

- $m+1$, if a patient seller arrives;

- $m-i, i=1, \ldots, k$ if an impatient $i$-buyer arrives.

In state $M$ there is some randomization: the bottom seller may leave after the first arrival of 
a Poisson process with intensity $\mu$.

Definition 3. Let $\mu \geq 0$ and $M>0$ an integer. A sequence $f_{m}, m=1, \ldots, M$, is called a $(\mu, M)$-chain if

$$
\left\{\begin{array}{l}
f_{0}=f_{-1}=\cdots=f_{1-k}=A \\
\left(\lambda+\sum_{i=1}^{k} \lambda_{i}\right) f_{m}+r=\lambda f_{m+1}+\sum_{i=1}^{k} \lambda_{i} f_{m-i} \\
\left(\sum_{i=1}^{k} \lambda_{i}+\mu\right) f_{M}+r=\left(\lambda_{1}+\mu\right) f_{M-1}+\sum_{i=2}^{k} \lambda_{i} f_{M-i}
\end{array}\right.
$$

The $(\mu, M)$-chain $f_{m}$ is called maximal if $f_{M}=B$.

Now one can put to use equation (18) to show the following result.

Proposition 7. A maximal $(\mu, M)$-chain $f_{m}$ is strictly decreasing in $m$ if $m<M$ and strictly increasing if $m>M$. It satisfies the formula

$$
\begin{gathered}
f_{m}=C_{0}+C_{1} \alpha_{1}^{m}+C_{2} \alpha_{2}^{m}+\cdots+C_{k} \alpha_{k}^{m}+a m, \quad \text { where } \\
a=\frac{r}{\lambda-\sum_{i=1}^{k} i \lambda_{i}}>0 \quad \text { and } \quad\left|\alpha_{1}\right|,\left|\alpha_{2}\right|, \ldots,\left|\alpha_{k}\right|<1
\end{gathered}
$$

The complex numbers: $\alpha_{0}=1, \alpha_{1}, \ldots, \alpha_{k}$ are the roots of the polynomial

$$
P(X)=\lambda X^{k+1}-\left(\lambda+\sum_{i=1}^{k} \lambda_{i}\right) X^{k}+\sum_{i=1}^{k} \lambda_{i} X^{k-i}
$$

Proof. Consider the middle equation in (19) for $m=M$ (by extending $f_{m}$ to be defined for $m=M+1)$. Subtracting that equation from the bottom one, one obtains

$$
\mu\left(f_{M-1}-f_{M}\right)=-\lambda\left(f_{M}-f_{M+1}\right)
$$

Equation (18) can be used to show that the sequence $\phi_{m}=f_{m}-f_{m+1}$ is always decreasing in $m$. The above equation shows that $\phi_{m}$ changes sign at $M$.

Now the middle equation in (19) is a difference equation that has general solution $f_{m}=$ $f_{m}^{0}+C_{0} \alpha_{0}^{m}+C_{1} \alpha_{1}^{m}+\cdots+C_{k} \alpha_{k}^{m}$, where $f_{m}^{0}$ is a particular solution, and $\alpha_{0}=1, \alpha_{1}, \ldots, \alpha_{k}$ are the roots of $P(X)$ (which is the polynomial corresponding to the recursive equation). If one tries a particular solution $f_{m}^{0}=a m$, one gets that indeed $a=\frac{r}{\lambda-\sum_{i=1}^{k} i \lambda_{i}}$. Moreover, if 
one defines $Q(X)=P(X) /(X-1)$, the roots of $Q(X)$ are $\alpha_{1}, \ldots, \alpha_{k}$. One calculates

$$
Q(X)=\lambda X^{k}-\left(\lambda_{1}+\cdots+\lambda_{k}\right) X^{k-1}-\cdots-\left(\lambda_{k-1}+\lambda_{k}\right) X-\lambda_{k} .
$$

For each $i=1, \ldots, k$, consider the equation $Q\left(\alpha_{i}\right)=0$, which is the same as $\lambda=\left(\lambda_{1}+\right.$ $\left.\cdots+\lambda_{k}\right) \alpha_{i}^{-1}+\cdots+\left(\lambda_{k-1}+\lambda_{k}\right) \alpha_{i}^{-(k-1)}+\lambda_{k} \alpha_{i}^{-k}$. Suppose $\left|\alpha_{i}\right| \geq 1$. Then using the previous equation one gets $\lambda \leq\left(\lambda_{1}+2 \lambda_{2}+\ldots+k \lambda_{k}\right)<\lambda$, contradiction. This implies that $\left|\alpha_{i}\right|<1$.

The description of the equilibrium is the following:

Theorem 8. Given $A, B, r, \lambda$ and $\lambda_{i}, i=1, \ldots, k$ which satisfy the inequalities above, there exists a unique competitive stationary Markov equilibrium of the game. Denote by $i_{0}=$ $\min \{k, m\}$. In equilibrium there are at most $M$ limit orders in the book, and if $i=1, \ldots, i_{0}$, then the level of the $i$ 'th limit order (counted from bottom up) in state $m<M$ is given by

$$
a_{i}(m)=\frac{\lambda_{k} f_{m-k}+\lambda_{k-1} f_{m-k+1}+\ldots+\lambda_{i} f_{m-i}}{\lambda_{k}+\lambda_{k-1}+\ldots+\lambda_{i}}
$$

where by convention $f_{0}=f_{-1}=\cdots=f_{1-k}=A$. The value function in state $m$ is given by $f_{m}$. The strategy of each agent in state $m$ is the following:

- If $m=1$, then place a limit order at $a_{1}(1)=A$.

- If $m=2, \ldots, M-1$, look at the bottom $k$ levels (or at all $m$ levels if $m<k$ ), which are $a_{1}(m), \ldots, a_{i_{0}}(m)$. If any of them is not occupied, occupy it. Anything above $a_{i_{0}}(m)$ does not matter.

- If $m=M$, the strategy is the same as for $m=2, \ldots, M-1$, except for the bottom seller at $a_{M}$, who exits (by placing a market order at B) after the first arrival in a Poisson process with intensity $\mu$.

- If $m>M$, then immediately place a market order at $B$.

One can make these formulas more explicit. There are two cases, depending on whether the $k$-unit market orders clear all the limit orders in the book or not. 
Case 1: $m \geq k$.

$$
\begin{aligned}
a_{m} & =f_{0}, \\
a_{m-1} & =f_{1} \\
& \vdots \\
a_{k+1} & =f_{m-k-1}, \\
a_{k} & =f_{m-k}, \\
a_{k-1} & =\frac{\lambda_{k} f_{m-k}+\lambda_{k-1} f_{m-k+1}}{\lambda_{k}+\lambda_{k-1}}, \\
a_{k-2} & =\frac{\lambda_{k} f_{m-k}+\lambda_{k-1} f_{m-k+1}+\lambda_{k-2} f_{m-k+2}}{\lambda_{k}+\lambda_{k-1}+\lambda_{k-2}}, \\
& \vdots \\
a_{1} & =\frac{\lambda_{k} f_{m-k}+\lambda_{k-1} f_{m-k+1}+\cdots+\lambda_{1} f_{m-1}}{\lambda_{k}+\lambda_{k-1}+\cdots+\lambda_{1}},
\end{aligned}
$$

Note that the levels $a_{k+1}, \ldots, a_{m}$ were chosen by convention, since it does not matter what happens above $a_{k}$.

Case 2: $m<k$.

$$
\begin{aligned}
a_{m} & =\frac{\sum_{i=m}^{k} \lambda_{i} A}{\sum_{i=m}^{k} \lambda_{i}}=A, \\
a_{m-1} & =\frac{\sum_{i=m}^{k} \lambda_{i} A+\lambda_{m-1} f_{1}}{\sum_{i=m}^{k} \lambda_{i}+\lambda_{m-1}}, \\
& \vdots \\
a_{1} & =\frac{\sum_{i=m}^{k} \lambda_{i} A+\lambda_{m-1} f_{1}+\cdots+\lambda_{1} f_{m-1}}{\sum_{i=m}^{k} \lambda_{i}+\lambda_{m-1}+\cdots+\lambda_{1}} .
\end{aligned}
$$

\section{Price Impact of Transactions}

Having now a tractable model of the limit order book, one can meaningfully talk about price impact in this model. For example, suppose the system is in state $m$ and a buyer submits a market order for one unit. Then the system moves automatically to state $m-1$ (there is one less seller). The ask price therefore moves from $a_{m}$ to $a_{m-1}$. If the buyer wants now to purchase another unit, then he must do so at a different price. 


\subsection{The Shape of the Limit Order Book}

In this model, agents stay on different levels in the book even though they are identical. They do this because they are indifferent between staying at one level and switching on another level (as long as the other agents stay on the same levels). The key assumption here is that some market orders may arrive, even with very small probability. Then it may be optimal for agents to cluster away from the ask, to capture the incoming multi-unit market orders. In fact, one can argue that what matters here are the expectations that traders have about the arrival rates of the incoming market orders, and not the actual values. Suppose the agents expect that large market orders will arrive. Then they will stay at higher levels than they would normally do. Therefore, one must keep in mind that the values $\lambda_{1}, \ldots, \lambda_{k}$ can be interpreted as representing what agents expect about incoming market orders. When one analyzes the shape of the price impact function, it turns out that a crucial factor is how fast the rates $\lambda_{i}$ decrease.

To quantify price impact, I use the theoretical results obtained in Section 3.3. Recall that $\lambda_{i}$ is the arrival rate of $i$-unit impatient sellers $(i=1, \ldots, k)$, and $\lambda>\sum_{i=1}^{k} i \lambda_{i}$ is the arrival rate of patient sellers. To get some intuition, suppose $\lambda_{1}$ is larger than the rest. For example, consider a function $\phi(i)$ which is decreasing in $i$, and some small value $\lambda_{0}>0$ such that

$$
\lambda_{1}=1 \quad \text { and } \quad \lambda_{i}=\lambda_{0} \phi(i), \text { if } i \geq 2 .
$$

Then set $\lambda=\sum_{i=1}^{k} i \lambda_{i}$ (plus some small number, so that one has indeed $\lambda>\sum_{i=1}^{k} i \lambda_{i}$; in fact it can be equal, it does not change the analysis). The problem I want to investigate is to calculate the price impact function for different choices of $\phi(i), i=2, \ldots, k$. Take for example

$$
\phi_{1}(i)=\frac{1}{i(i+1)}, \quad \phi_{2}(i)=\frac{4}{2^{i}}
$$

The price impact function in state $m$ is defined as the change in the ask price when $i$ units are bought via a market order of $i$ units:

$$
\operatorname{Imp}(i, m)=a_{i+1}(m)-a_{1}(m), \quad \text { as a function of } i(\text { and } m) .
$$

To calculate the price impact function, one can apply the theoretical results of the previous 
section in the following way: Equation (19) shows that $f_{m}$ satisfies a recursive formula with initial conditions $f_{0}=f_{-1}=f_{1-k}=A$. This fixes $k$ coefficients of $f_{m}$ in equation (20). To fix the last coefficient, one should use the bottom equation in (19). For simplicity, I choose a different method: Let $B$ become a free parameter, and choose another parameter $\delta>0$, so that

$$
f_{1}=A-\delta
$$

This fixes all coefficients $C_{i}$, so one can determine $M$ as the first $m$ for which $f_{m}$ starts increasing (according to Proposition 7). Then one determines $B$ by $B=f_{M}$. So in the analysis that follows and in Figure 2, instead of $B$, I use the parameter $\delta$.

In Figure 2, I compare the graphs of the price impact function $\operatorname{Imp}(i, m)$ for the two functions $\phi_{1}=1 / i(i+1)$ and $\phi_{2}=4 / 2^{i}$. In both cases the maximum number of sellers is 41 (so $m<41$ ), and I display the results when the book has $m=10,20,30,40$ limit sell orders. All the graphs shown are for $k=20$, which means that the sellers in the book believe that market buy orders for more than 20 units appear with zero probability. In the first case (for $\left.\phi_{1}\right)$, the sellers believe that the arrival rate $\lambda_{i}$ of $i$-market orders does not decrease very fast in $i$, while in the second case they believe that the arrival rate decreases exponentially. The top four plots refer to $\phi_{1}$, and the bottom four to $\phi_{2}$.

Notice that in the case of $\phi_{1}$, the price impact function $\operatorname{Imp}(i)$ is concave when $i \leq k$ units are purchased (recall that $k$ is the maximum size of a market buy order that sellers in the book expect to occur). So when $m$ itself is less than $k$, the price impact function is concave everywhere, which is the case of the first two graphs. The intuition for this finding is the following: each seller above the ask up to level $k$ believes that his limit order will be cleared by a market order with a probability which is not too small. Then instead of clustering near the ask, they prefer to take advantage of the large market orders and cluster above the ask. This leads to a concave price impact function. Above the level $k$ or when the probability of a large market order decreases too fast, the price impact function is linear, and even convex. This is the case for all the graphs for $\phi_{2}$, and in the region where $i>k$ for $\phi_{1}$.

Overall, the conclusion seems to be that for smaller orders the price impact function should be mildly concave, and for larger orders it should be mildly convex. This reflects the existing differences of opinions in the literature, which has not said yet the final word whether the price impact is concave, linear, or convex, and in what range. 

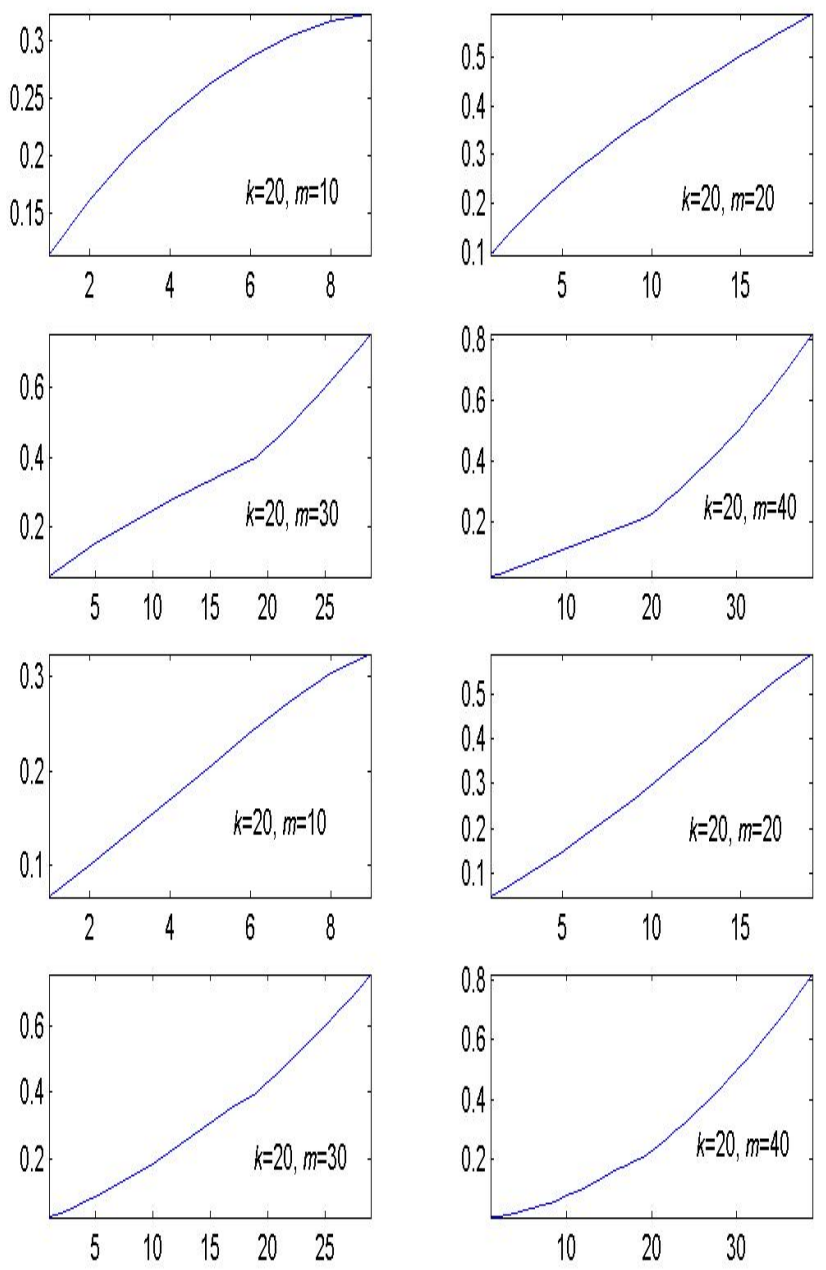

Figure 2: The instantaneous price impact function $\operatorname{Imp}(i)=a_{i+1}-a_{1}$ plotted against $i$. ( $\operatorname{Imp}(i)$ is the difference in the level of the $i+1$ 'st limit sell order above the ask and the ask price.) The values of parameters are $A=1, r=0.001, \delta=0.04, k=20$, and the arrival rates are $\lambda_{1}=1 ; \lambda_{i}=\lambda_{0} \phi(i), i=2, \ldots, k$, where $\lambda_{0}=10^{-5} ; \lambda=\sum_{i=1}^{k} i \lambda_{i}$. The top four plots correspond to the weight function $\phi(j)=1 / j(j+1)$; the bottom four plots to $\phi(j)=4 / 2^{j}$. Each set of four graphs is considered for the case when there are $m=10,20,30,40$ sell orders in the book. The number $k$ is the maximum number of units that market buy orders can have, and $\phi(i)$ indicates how fast the arrival rate of $i$-market orders decreases with $i$. For the top four plots (when $\phi(i)$ does not decrease too fast), notice the concave shape of the price impact function $\operatorname{Imp}(i)$ in the region where $i \leq k$. For the other regions notice either linearity or convexity of price impact. 


\subsection{Theoretical vs. Observed Price Impact}

In the preceding discussion about price impact, I introduced what might be called "theoretical" price impact, which represents the instantaneous reaction of the ask price to a large market order. When one estimates price impact from data, one should also take into account that agents who submit market orders may strategically want to divide the market order in smaller pieces. Then, the "observed" price impact might be different from the theoretical one.

To understand better this difference, I give an example set in the framework of Section 3.2 (with patient sellers arriving faster than impatient buyers). Suppose the system is in state $M$, and a strategic agent wants to place a market buy order for two units. Then the first market order for one unit will be placed immediately, and the system moves to $M-1$. Then a dilemma arises: should the agent place a market buy now for the price of $a_{M-1}$, or wait until the system goes again to state $M$ and place a market buy for $a_{M}<a_{M-1}$ ? That depends on the patience of the agent, and on the time the agent has to wait.

For this, one has to calculate the mean expected (first) passage time from state $M-1$ to $M: t_{M-1, M}$. In general, the mean passage time $t_{i j}$ from state $i$ to $j$ can be calculated from the following system of equations:

$$
t_{i j}=1 \cdot P_{i j}+\sum_{k \neq j}\left(1+t_{k j}\right) P_{i k}
$$

where $P$ is the Markov transition matrix (with states $0,1, \ldots, M$ ):

$$
P=\left[\begin{array}{ccccccc}
1-\omega & \omega & 0 & 0 & \cdots & 0 & 0 \\
1-\omega & 0 & \omega & 0 & \cdots & 0 & 0 \\
0 & 1-\omega & 0 & \omega & \cdots & 0 & 0 \\
0 & 0 & 1-\omega & 0 & \cdots & 0 & 0 \\
\vdots & \vdots & \vdots & \vdots & \ddots & \vdots & \vdots \\
0 & 0 & 0 & 0 & \cdots & 0 & \omega \\
0 & 0 & 0 & 0 & \cdots & 1-\omega & \omega
\end{array}\right]
$$

To calculate $t_{i j}$ in general, one looks at the column $t^{j}$ formed with $t_{i j}$. Let $\tilde{P}_{j}$ be the matrix obtained from $P$ by replacing the $j$ 'th column by zero. Let $e$ be the column vector formed 
with ones. Then $t^{j}$ can be obtained from the formula:

$$
t^{j}=e+\tilde{P}_{j} t^{j}
$$

In our case $t_{m, M}$ satisfies a recursive formula in $m$, and one calculates

$$
t_{m, M}=\beta(M-m)-\frac{\beta}{\alpha^{-1}-1}\left(\alpha^{m}-\alpha^{M}\right), \quad \alpha=\frac{1-\omega}{\omega}, \beta=\frac{1}{2 \omega-1} .
$$

So $t_{M-1, M}=\beta-\beta \alpha^{M} \approx \beta$. Now the mean passage time is calculated in units of time $=\frac{1}{\lambda_{1}+\lambda_{2}}$. Denote by $r_{0}$ the time discounting coefficient of the agent, and by

$$
\varepsilon_{0}=\frac{r_{0}}{\lambda_{1}+\lambda_{2}}
$$

The choice is then between $a_{M-1}$ and $a_{M}+\varepsilon_{0} \beta$. If the agent who places market orders can place limit orders instead, then the choice is between $f_{M-1}$ and $f_{M}+\varepsilon_{0} \beta$. But $f_{M-1}=f_{M}+u \varepsilon$. So the agent waits until $t_{M-1, M}$ if and only if $r u>r_{0} \beta$. I have assumed $s=0$ for simplicity (so in the state $M$ there is no mixing). Then the agent waits if and only if

$$
r_{0}<r \frac{2 \omega-1}{1-\omega}
$$

\section{Equilibrium: The General Case}

For simplicity, I analyze the general case, when all arrival rates are equal

$$
\lambda=\lambda_{P B}=\lambda_{P S}=\lambda_{I B}=\lambda_{I S}>0 .
$$

Later on, I indicate how similar results can be obtained when the arrival rates are different (the most important case is when $\lambda_{1}=\lambda_{P B}=\lambda_{P S}$ is larger than $\lambda_{2}=\lambda_{I B}=\lambda_{I S}$ ).

To get some intuition about the equilibrium, consider a setup similar to that of the onesided case, but suppose that after after a patient seller (which has a limit sell order at $A$ ) a patient buyer arrives to the market. Then the buyer behaves as a monopolist towards the potential incoming impatient sellers, and places a limit buy order at $B$. In this situation, if the reservation value of the seller is larger than the reservation value of the buyer, they will 


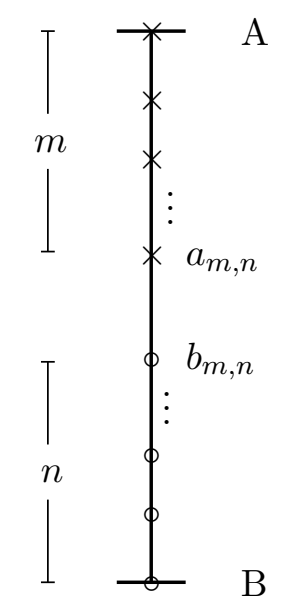

Figure 3: The limit order book with $m$ sellers and $n$ buyers.

not be tempted to make offers to one another, and would rather wait to trade with future impatient agents (this happens for most values of the parameters of the model). It follows that patient buyers and sellers behave very much like in the one-sided case, where new patient agents just keep placing bid-ask improving limit orders until it is better to trade immediately rather than wait. Thus, patient agents form two queues, a descending one starting from $A$, and an ascending one starting from $B$ (see Figure 3 below). However, at some point, the two queues get close to each other, and the patient traders at the bid and the ask may want to trade with each other immediately instead of waiting for impatient agents to place market orders. In this case, I say that the limit order book is full (or saturated). To get a quantitative solution of this problem, one must describe more precisely when the book becomes full.

\subsection{Theory}

As in the one-sided case, I start by assuming that the system is already in equilibrium, and try to find necessary conditions. This is the difficult part, and at the first reading one may want to skip to the second part of Theorem 9, which is the one most useful for applications. The second part of the Theorem says that, given the solution of some system of partial difference equations in the plane, one can construct a stationary Markov perfect equilibrium (MPE) of the game. This allows one to give a lot of examples of an equilibrium limit order book. However, if one wonders in what sense the equilibrium is unique, the following analysis is 


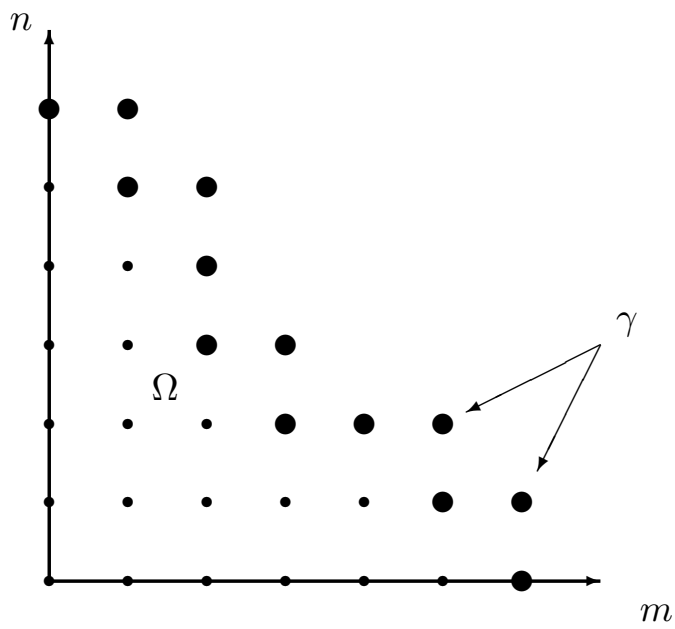

Figure 4: The state region $\Omega$ and the boundary $\gamma$.

important.

So assume that the system has reached a competitive, stationary Markov perfect equilibrium. (See Appendix B for definitions. The Markov structure is given by the pair $(m, n)$ of the number of sellers and buyers.) In state $(m, n)$ at time $t$, denote by $f_{m, n}$ the expected utility of the sellers and by $g_{m, n}$ the expected utility of the buyers (they do not depend on $t$ because this is a stationary equilibrium). By definition

$$
f_{m, n}=\max _{\tau} \mathrm{E}_{t}\left\{P^{s}(\tau)-r(\tau-t)\right\}
$$

where $P^{s}(\tau)$ is the selling price at the stopping time $\tau$. Similarly,

$$
g_{m, n}=\min _{\tau} \mathrm{E}_{t}\left\{P^{b}(\tau)+r(\tau-t)\right\}
$$

where $P^{b}(\tau)$ is the buying price at $\tau$ (recall that $g_{m, n}$ represents minus the utility of the buyers in state $(m, n))$. Define by $a_{m, n}$ the ask price, and by $b_{m, n}$ the bid price.

Definition 4. A state is $(m, n)$ is called regular if in equilibrium traders stay for an expected positive time. Define the state region $\Omega$ as the collection of all regular states. A state in which some agent can exit via a mixed strategy is called partial. If the mixing behavior happens as in Corollary 20, I call the partial state rigid (this means that mixing is done only 
by the bottom agent). The boundary $\gamma$ of $\Omega$ is the set of partial states. A state in which the system stays for zero time is called fleeting.

Also, a point in $\Omega$ not on $\gamma$ is called "interior." Proposition 21 implies that all interior points represent states in which agents wait until the arrival of a new agent. As shown in Proposition 21, the partial states only appear when the book becomes full, so partial states will indeed lie on the geometric boundary $\gamma$ (see Figure 4).

Now Proposition 21 shows that if one starts at each point in $\Omega$ and goes along the main diagonal, there is at most one partial state on the boundary $\gamma$. If for all starting points in $\Omega$, the corresponding boundary state is partial rigid, I call the equilibrium rigid. In words, having a rigid equilibrium means that when the limit order book becomes full, traders always use mixed strategies; moreover, mixing is done in such a way so that all agents have the same value function. (Using Corollary 20, one can show that in a partial rigid state $(m, n)$ the value functions for the buyers and sellers are equal: $f_{m, n}=g_{m, n}$. This in fact is the main reason why I use rigid partial states.)

From now on I assume the system has reached a rigid competitive stationary MPE. Then the resulting state space satisfies Lemma 18, and this implies the existence of a non-increasing shape function $\phi$ that completely characterizes $\Omega$. Using the shape $\phi$, one can show that the points in $\Omega$ can only be of one of the types described in Figure 5 (use also Corollary 21). (In principle, there is one more type of boundary point, where the shape function $\phi$ takes the value zero more than once, but it is easy to show that this behavior cannot appear in equilibrium.)

Now, as in the one-sided case, it is a good idea to find a recursive structure for the value functions $f$ and $g$. From state $(m, n)$ the system can go to the following neighboring states:

- $(m-1, n)$, if an impatient buyer arrives; also, if a patient buyer arrives and submits a fleeting limit order;

- $(m+1, n)$, if a patient seller arrives and submits a (non-fleeting) limit order;

- $(m, n-1)$, if an impatient seller arrives; also, if a patient seller arrives and submits a fleeting limit order;

- $(m, n+1)$, if a patient buyer arrives and submits a limit order;

- $(m-1, n-1)$, if after a positive expected time in state $(m, n)$ a pair of existing patient traders, a buyer and a seller, trade with each other via a fleeting limit order. 


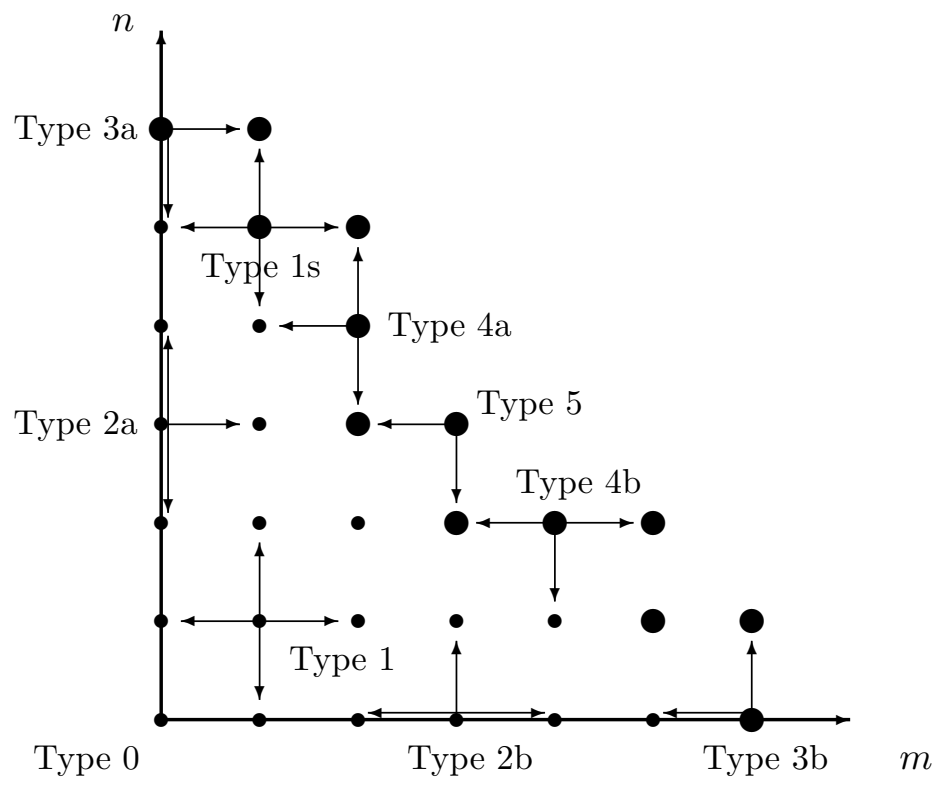

Figure 5: Types of points in the state region $\Omega$.

From an interior state $(m, n)$ (of Type 1 ) the system can go only to the states $(m-1, n)$, $(m+1, n),(m, n-1)$, or $(m, n+1)$. The arrival of the first of these four states happens after a random time, which is exponentially distributed with parameter $4 \lambda$. Then each event happens with probability $1 / 4$. One obtains the formula $f_{m, n}=\frac{1}{4}\left(f_{m-1, n}+f_{m+1, n}+f_{m, n-1}+\right.$ $\left.f_{m, n+1}\right)-r \cdot \frac{1}{4 \lambda}$. Denote again by

$$
\varepsilon=\frac{r}{\lambda}
$$

The formula becomes

$$
4 f_{m, n}+\varepsilon=f_{m-1, n}+f_{m+1, n}+f_{m, n-1}+f_{m, n+1} .
$$

This type of reasoning works for all the other states in $\Omega$.

I now describe the equilibrium. The relevant parameters are $A, B$ and $\varepsilon=r / \lambda$, i.e., the ratio between the time discount coefficient and the arrival intensity.

Theorem 9. Consider a competitive stationary Markov equilibrium of the game with both buyers and sellers, where all types of traders arrive at equal rates. Assume that the equilibrium is also rigid. Then there exists a state region $\Omega$ as described in Lemma 18, and drawn in 
Figure 5. For a state $S=(m, n)$ in $\Omega$, the corresponding value functions $f$ and $g$ satisfy the following equations:

- If $S$ is of Type 0 ,

$$
\begin{aligned}
& f_{0,0}=A \\
& g_{0,0}=B
\end{aligned}
$$

- If $S$ is of Type 1 ,

$$
\begin{aligned}
& 4 f_{m, n}+\varepsilon=f_{m-1, n}+f_{m+1, n}+f_{m, n-1}+f_{m, n+1} \\
& 4 g_{m, n}-\varepsilon=g_{m-1, n}+g_{m+1, n}+g_{m, n-1}+g_{m, n+1}
\end{aligned}
$$

- If $S$ is of Type $1 s$, then for some $s_{m, n} \geq 0$,

$$
\begin{gathered}
\left(4+s_{m, n}\right) f_{m, n}+\varepsilon=f_{m-1, n}+f_{m+1, n}+f_{m, n-1}+f_{m, n+1}+s_{m, n} f_{m-1, n-1}, \\
\left(4+s_{m, n}\right) g_{m, n}-\varepsilon=g_{m-1, n}+g_{m+1, n}+g_{m, n-1}+g_{m, n+1}+s_{m, n} g_{m-1, n-1}, \\
f_{m, n}=g_{m, n}
\end{gathered}
$$

- If $S$ is of Type 2a,

$$
\begin{gathered}
f_{0, n}=A \\
3 g_{0, n}-\varepsilon=g_{0, n-1}+g_{0, n+1}+g_{1, n} ;
\end{gathered}
$$

and similarly for Type $2 b$.

- If $S$ is of Type $3 a$,

$$
\begin{gathered}
f_{0, n}=A \\
\left(2+s_{0, n}\right) g_{0, n}-\varepsilon=\left(1+s_{0, n}\right) g_{0, n-1}+g_{1, n} ;
\end{gathered}
$$

and similarly for Type $3 b$.

- If $S$ is of Type $4 a$, then for some $s_{m, n} \geq 0$,

$$
\begin{gathered}
\left(4+s_{m, n}\right) f_{m, n}+\varepsilon=f_{m-1, n}+2 f_{m, n-1}+f_{m, n+1}+s_{m, n} f_{m-1, n-1}, \\
\left(4+s_{m, n}\right) g_{m, n}-\varepsilon=g_{m-1, n}+2 g_{m, n-1}+g_{m, n+1}+s_{m, n} g_{m-1, n-1}, \\
f_{m, n}=g_{m, n}
\end{gathered}
$$

and similarly for Type $4 b$.

- If $S$ is of Type 5, then for some $s_{m, n} \geq 0$,

$$
\begin{gathered}
\left(4+s_{m, n}\right) f_{m, n}+\varepsilon=2 f_{m-1, n}+2 f_{m, n-1}+s_{m, n} f_{m-1, n-1} \\
\left(4+s_{m, n}\right) g_{m, n}-\varepsilon=2 g_{m-1, n}+2 g_{m, n-1}+s_{m, n} g_{m-1, n-1} \\
f_{m, n}=g_{m, n}
\end{gathered}
$$

Conversely, for each solution of the system above with $s_{m, n} \geq 0$ and $f_{m, n} \geq g_{m, n}$ there exists a corresponding rigid competitive stationary Markov equilibrium. 
Proof. See Appendix A.

I call the numbers $s_{m, n}$ slack variables. They equal $s_{m, n}=\mu_{m, n} / \lambda$, where $\mu_{m, n}$ is the Poisson exit rate in the partial state $(m, n)$ on the boundary. The direct implication of Theorem 9 is useful mainly for questions relating uniqueness of equilibria (so far, it seems that equilibria are very close to each other, or perhaps even unique). However, the more important implication is the converse one. Suppose there is a region $\Omega$ of the type mentioned above, such that for all $(m, n) \in \Omega$ there exist numbers $f_{m, n}, g_{m, n}, s_{m, n}$ which satisfy the equations of Theorem 9. Then the Theorem guarantees the existence of an equilibrium of the game. To understand what kind of strategies give this equilibrium, one needs to define two set of numbers $b_{m, n}$ (bid prices), and $a_{m, n}$ (ask prices) for each $(m, n) \in \Omega$.

Proposition 10. Consider a competitive stationary Markov equilibrium as in Theorem 9, and let $S=(m, n)$ be a state in $\Omega$. Then the corresponding bid price $b_{m, n}$ and ask price $a_{m, n}$ satisfy

- If $S$ is of Type 1,

$$
\begin{aligned}
& a_{m, n}=f_{m-1, n}, \\
& b_{m, n}=g_{m, n-1} ;
\end{aligned}
$$

- If $S$ is of Type 2a,

$$
\begin{gathered}
a_{0, n}=A, \\
b_{0, n}=g_{0, n-1} ;
\end{gathered}
$$

and similarly for Type $2 b$.

- If $S$ is of Type 5, then for some $s_{m, n} \geq 0$,

$$
\begin{aligned}
& a_{m, n}=\left(1+s_{m, n}\right) f_{m-1, n}-s_{m, n} f_{m, n}, \\
& b_{m, n}=\left(1+s_{m, n}\right) g_{m, n-1}-s_{m, n} g_{m, n}
\end{aligned}
$$

The formulas for the other types of boundary points are similar.

I now briefly describe the corresponding equilibrium strategies. Let $(m, n)$ be a state which does not necessarily belong to $\Omega$ (one must also describe what happens in the fleeting states). Then define the following strategy profile: 
- If $(m, n)$ is in $\Omega$ but not on the boundary $\gamma$, each seller plays the strategy: place a limit sell order at $a_{m, n}$. The seller can also place the limit order at any point above, but in that case some other seller must have a limit order at $a_{m, n}$; otherwise, immediately drop to $a_{m, n}$. The strategy works in a similar way for buyers and $b_{m, n}$.

- If $(m, n)$ is in $\gamma$, let $\mu_{m, n}=\lambda s_{m, n}$. Then the strategy is similar to the one above, except that the seller at the ask may randomly change the limit order from $a_{m, n}$ to $f_{m, n}$ at a Poisson rate of $\mu_{m, n}$, and the buyer at the bid will immediately accept by placing a market order.

- If $(m, n)$ is a fleeting state with $m, n>0$, then define the value functions $f_{m, n}$ and $g_{m, n}$ by looking at the values from the corresponding state on $\gamma$ along the main diagonal. (If the main diagonal does not intersect $\gamma$, but one of the coordinate axes, simply define $f_{m, n}=g_{m, n}$ to be either $A$ or $B$ depending on whether it is the $x$-axis or the $y$-axis, respectively.) Then the strategy for each seller is to place a limit order at $f_{m, n}$ and for some buyer to instantly accept it by placing a market order. Which order gets executed is determined randomly.

- If $(m, n)$ is a fleeting state with $n=0$, define $f_{m, n}=g_{m, n}=B$. Then the strategy for each seller is to place a market order at $B$ (so that only one of them is executed, at random). A similar description is for fleeting states $(m, n)$ with $m=0$.

Given the equations that all these numbers satisfy, it is not hard to see that the strategy profile just defined is an SPE, and indeed a competitive stationary Markov equilibrium.

Coming back to the expression for the equilibrium in Theorem 9 one can see that both $f_{m, n}$ and $g_{m, n}$ satisfy some finite difference equations, so one may ask if in the limit, when $\varepsilon$ is very small, whether $f$ and $g$ do not satisfy some differential equations. It turns out that the answer is yes. Let $\varepsilon=\delta^{2}, x=m \delta$ and $y=n \delta$. Define the functions $f$ and $g$ at the discrete values $(x, y)=(m \delta, n \delta)$ by

$$
f(x, y)=f_{m, n}, \quad g(x, y)=g_{m, n}
$$

Then one gets the following asymptotic result: 
Theorem 11. The solution of the model converges when $\varepsilon=r / \lambda$ is small to the solution of the following system of partial differential equations with a free boundary $\gamma$ :

$$
\left\{\begin{array} { l } 
{ \triangle f = 1 , } \\
{ f ( 0 , y ) = A , } \\
{ \frac { \partial f } { \partial y } ( x , 0 ) = 0 , } \\
{ \frac { \partial f } { \partial x } + \frac { \partial f } { \partial y } = 0 \text { at } \gamma ; }
\end{array} \quad \left\{\begin{array}{l}
\triangle g=-1, \\
g(x, 0)=B, \\
\frac{\partial g}{\partial x}(0, y)=0, \\
\frac{\partial g}{\partial x}+\frac{\partial g}{\partial y}=0 \text { at } \gamma ;
\end{array}\right.\right.
$$

where the free boundary $\gamma$ is determined by the condition

$$
f=g \text { at } \gamma
$$

The problem has a unique solution, which is symmetric in $x$ and $y$. The curve $\gamma$ is slightly concave-down, and passes approximately through the points $(2,0)$ and $(0,2)$.

Proof. See Appendix A.

This is a Poisson equation in a closed region with mixed-derivative conditions at the boundary. The condition $f=g$ at the boundary determines the free boundary $\gamma$, where the limit book is saturated. Since the oblique derivative is never tangent to $\gamma$, the problem is well posed, and one can write an algorithm to solve it, using for example finite differences.

\section{$5.2 \quad$ Numeric Results}

Having described the equilibrium, one may wonder how to actually find it. This is not a trivial problem: for each value of $A, B$ and $\varepsilon$, one needs to find the state region $\Omega$ (or equivalently the boundary $\gamma$ ) and solve the system given in Theorem 9 . One could take the brute force approach and for each $\Omega$ within reasonable values try to solve the system. For $\varepsilon$ small however, the complexity of this approach becomes daunting. The reason is that this is a system of equations with a free boundary $\gamma$, and one needs to simultaneously find the solution of the system and the shape of the boundary.

To find the state region $\Omega$, one uses the intuition coming from the asymptotic result in Theorem 11. One sees that the asymptotic boundary is slightly concave, so it is a good idea to try regions state regions $\Omega$ which are close to being triangular (bounded by the coordinate axes and the line $X+Y=R$ ). As one increases the size of $\Omega$, one will be forced to take out a 
Table 1: Solution in the general case with both buyers and sellers, for $A=1, B=0, \varepsilon=0.09$. Left bottom corner corresponds to state $(0,0)$. The number in position $(m, n)$ represents the value function $f_{m, n}$ for the sellers in state $(m, n)$. The shape function is $\phi=[0,1,1,2,2,2,3]$, which leads to the state region $\Omega$ as in Figure 4 . The vector $s$ collects the slack variables along the boundary, starting from $(0,6)$ down to $(6,0)$. The value function $g_{m, n}$ for the buyers is given by the formula $g_{m, n}=1-f_{n, m}$. The bullets in positions $(3,4)$ and $(4,3)$ (which are not in $\Omega$ ) indicate the departure of the shape of $\Omega$ from the triangular one.

$$
\begin{array}{rrrrrrr}
1.000 & 0.965 & & & & \\
1.000 & 0.905 & 0.824 & & & & \\
1.000 & 0.828 & 0.726 & \bullet & & & \\
1.000 & 0.770 & 0.616 & 0.500 & \bullet & & \\
1.000 & 0.726 & 0.526 & 0.384 & 0.274 & 0.176 & \\
1.000 & 0.697 & 0.468 & 0.300 & 0.177 & 0.095 & 0.035 \\
1.000 & 0.682 & 0.440 & 0.260 & 0.131 & 0.045 & 0.000
\end{array}
$$

few points from the triangle; otherwise, there would be no solution to the system of equations. Indeed, in the examples I computed, the shape of $\Omega$ is close to being triangular only when $\varepsilon$ is relatively large. As $\varepsilon$ gets smaller, points on the diagonal $X+Y=R$ start missing (see Table 1).

I revert now to the more general case when $\lambda_{1}=\lambda_{P B}=\lambda_{P S}$ may be different from $\lambda_{2}=\lambda_{I B}=\lambda_{I S}$. Define the parameter $\omega$ as the percentage of patient traders that arrive to the market:

$$
\omega=\frac{\lambda_{1}}{\lambda_{1}+\lambda_{2}}
$$

So far I only discussed the case $\omega=1 / 2$. But the case when $\omega>1 / 2$ is even more important. First, it corresponds to empirical evidence (see Biais et al. (1995)), which shows that more limit orders than market orders arrive to the market. ${ }^{19}$ And second, $\omega>1 / 2$ has the desirable

\footnotetext{
${ }^{19}$ The same empirical research indicates that when the spread is wider the percentage of submitted limit orders increases. However, for technical reasons I do not model spread-dependent arrival intensities.
} 
implication that there is pressure for spreads to revert to small values. If instead impatient agents arrived faster, then there would pressure for spreads to widen towards the value $A-B$.

When patient traders arrive faster than impatient traders, it is reasonable to expect that the waiting costs of patient agents increase. That implies that the limit order book will be

more "rarefied" than in the case $\omega=1 / 2$. The first guess is that the regions $\Omega$ for which one can find solutions are more concave than for $\omega=1 / 2$. Indeed this is the case, as can be seen numerically. This is consistent with the limiting case $\omega=1$ from the next Section, where I show that, if only patient people arrive to the market, the region $\Omega$ collapses to the coordinate axes.

\section{Equilibrium: The Homogeneous Case}

An interesting case to study is when all agents are equally patient $\left(\lambda_{I S}=\lambda_{I B}=0\right)$. For simplicity, I also assume that the arrival rates of patient sellers and patient buyers are equal:

$$
\lambda=\lambda_{P S}=\lambda_{P B}>0
$$

It turns out that in this case the limit order book cannot accommodate both buyers and sellers. The reason this happens is because traders lose their incentive to wait. In the one-sided case, sellers were waiting to extract rents from impatient buyers. Now, when all agents are equally patient, they cannot extract rents from each other, so a bargaining game follows. In principle, there can be many equilibria, unless one puts more structure on the bargaining problem. I do not follow this path here. Instead, I give an example of a competitive stationary Markov equilibrium, which seems to be the prototype for all such equilibria. For simplicity, assume $A=1, B=0$ (this can be done without any loss of generality). Define as before $\varepsilon=r / \lambda$.

As in the general case from the previous Section, one can talk about the state region. In order to describe an equilibrium, one also has to describe what happens in each state $(m, n)$.

Definition 5. Let $M, \mu, f_{m}, a_{m}$ be as in the Theorem 3 applied to $A=\frac{1}{2}$ and $B=0$ (for the bottom half of the limit order book). Define also $g_{m}=1-f_{m}$ and $b_{m}=1-a_{m}$, the symmetric values with respect to $1 / 2$. For $m>M$ extend $f_{m}=0$ and $g_{m}=1$. Let $\Omega$ be the collection of points $(0, m)$ and $(m, 0)$ for $m=0, \ldots, M$. 


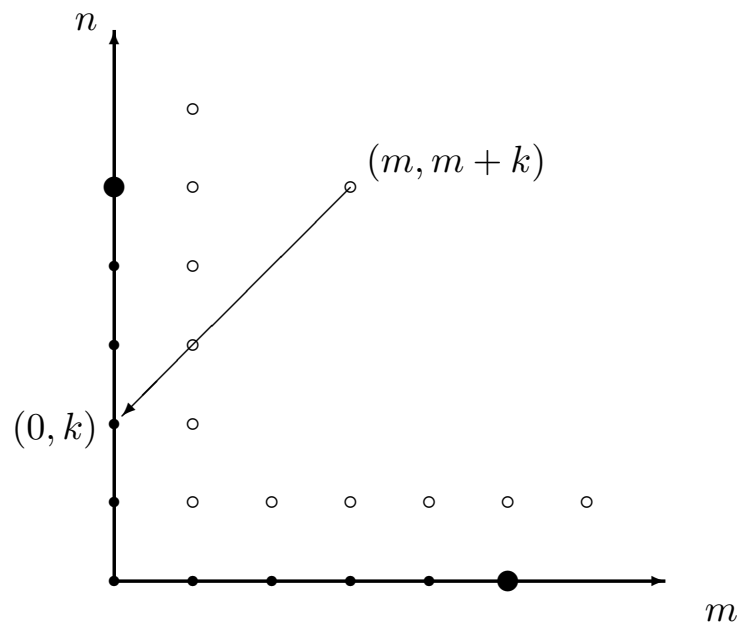

Figure 6: The state region in the homogeneous case. (Surrounding fleeting states are marked with a circle).

Theorem 12. Consider any state $(m, n)$ in the plane. Then a competitive stationary Markov equilibrium of the game is given by the following strategy profile:

- If $m=0$ or $n=0$, apply the same strategy as in the one-sided case. For example, in state $(0, n)$ with $0<n<M$ at least one buyer places a limit buy order at $b_{n}$ (and expects that an incoming seller will place a market order).

- If $m>n>0$, place immediately a limit buy (sell) order at $f_{m-n}$ (and expect that some seller (buyer) will immediately accept it).

- If $n>m>0$, place immediately a limit buy (sell) order at $g_{n-m}$.

- If $m=n>0$, place immediately a limit sell (buy) order at $f_{0}=g_{0}=1 / 2$.

Note that in each state $(m, n)$ with $m, n>0$, one has $f_{m, n}=g_{m, n}$. This is because the offers are made at this level, so those who exit get $f_{m, n}$ in expectation. Also, those who did not get the offer, move to the state $(m-1, n-1)$ on the same diagonal, where the value functions are the same as in state $(m, n)$.

Proof. Using the one-deviation property, assume that in all other states except $(m, n)$ agents behave as mentioned above, and show that for each agent behavior in state $(m, n)$ is optimal. 
If $(m, n)$ lies above or below the main diagonal, the proof is straightforward. The interesting case is on the diagonal, for example if $(m, n)=(1,1)$. Then as in Proposition 19, define $f_{1,1}^{\infty}$ the expected payoff of the sellers if they all wait, and by $g_{1,1}^{\infty}$ the same notion for the buyers. One calculates

$$
f_{1,1}^{\infty}=\frac{1}{2}\left(f_{1,2}+f_{2,1}\right)=\frac{1}{2}\left(g_{1}+f_{1}\right)=\frac{1}{2}
$$

since $f_{1}+g_{1}=1$ (by definition $f$ and $g$ were chosen symmetric with respect to $\frac{1}{2}$ ). Then the discounted value to time zero is $f_{1,1}^{0}=f_{1,1}^{\infty}-\frac{\varepsilon}{2}$ for the sellers, and $g_{1,1}^{0}=g_{1,1}^{\infty}+\frac{\varepsilon}{2}$ for the buyers. Since $f_{1,1}^{0}<g_{1,1}^{0}$, it follows that both the buyer and the seller prefer not to wait, and would make each other an offer in the interval $\left[\frac{1}{2}-\frac{\varepsilon}{2}, \frac{1}{2}+\frac{\varepsilon}{2}\right]$. In particular $f_{0}=g_{0}=\frac{1}{2}$ belongs to this interval.

Notice that other offers can be made in that interval, and it depends on agents beliefs. That suggests that the equilibrium is not unique. However, one can show that it is essentially unique. Recall that a rigid equilibrium has all boundary states partial rigid, i.e., in those states only the bottom traders use mixed strategies - which implies that the value functions are equal for the buyers and the sellers.

Proposition 13. Any rigid equilibrium in the homogeneous case must collapse onto the coordinate axes.

Proof. The boundary of the state region is by definition formed only with partial rigid states, where $f=g$. Now from a state $(m, n)$ the system can only go to $(m, n+1)$ or $(m+1, n)$, so $2 f_{m, n}+\varepsilon=f_{m+1, n}+f_{m, n+1}$, which means that $f$ keeps increasing as it moves closer to the boundary. Similarly, $g$ is decreasing towards the boundary. But at the boundary $f=g$, so in state $(m, n)$ it must have been that $f<g$, which means that it is not optimal for agents to wait in state $(m, n)$. So there are no states in $\Omega$ except for the partial ones. But then the same argument can be used for a partial state, so it is not optimal to wait there either.

\section{Empirical Implications}

\subsection{Market Orders and the Spread}

An interesting implication of the equilibrium in the general case is that a market sell order leads to an decrease in both the bid and the ask, but the decrease in the bid is larger. The 
intuition for this is given in Proposition 16: the departure of one limit buyer makes the buyers better off, and the sellers worse off (because of future possibility of trading with each other when the book becomes full). But that possibility is remote enough so that the decrease in the value function of the sellers is less than the decrease in the value function of the buyers (recall that the value function is minus the utility for the buyers). This intuition also appears in the analysis of the one-sided case, cf. equation (9).

This implication was noticed by Biais, Hillion and Spatt (1995), in their analysis of the order flow in the Paris Bourse. Their analysis goes as follows: "The downward shift in the book has two components, the decrease in the bid, merely reflecting that the large sale consumed the liquidity offered at that quote, and the subsequent decrease in the ask, reflecting the reaction of the market participants to the large sale. The decrease in the bid could be a transient decrease in the liquidity on this side of the book, or a permanent information adjustment. In our onelag analysis, we cannot differentiate the two hypotheses. In contrast to the behavior of the bid, the decrease in the ask is likely to reflect the information effect."

I argue that the decrease in the ask need not reflect an information effect. Indeed, it can simply be regarded as an adjustment made by the limit sellers, who, after the bid decreased, realize that they now have to wait more to execute their orders, and lower their offers accordingly. It is however quite hard to distinguish between the waiting costs story and the information story.

One may still argue that if information acquisition is not homogeneous across agents, there is actually a way of distinguishing between the two stories. Namely, consider the information story. If a large market sell order occurs, and this conveys information, then it is reasonable to expect more new agents than current ones to take advantage of the change. This is because it is presumably harder for current sellers in the book to change their beliefs about the value of the asset. Also, they may prefer not to cancel their limit orders, since this operation involves some costs (private and sometimes financial). So in the information story one should expect more new agents to arrive, and fewer limit order cancellations. This means that, if market sell orders are followed by a lot of cancellations on the ask side of the book, then that would reinforce more the waiting costs story.

And it turns out that this is true: cancellations on the ask side of the book are particularly frequent after market sell orders. This in fact is one of the strongest findings of Biais et al. 
besides the diagonal effect (which is just serial correlation of different types of orders). They suggest a possible explanation based on hidden orders in the book at the Paris Bourse, but as I explained above, the waiting costs story seems to be a likelier explanation.

\subsection{The Distribution of the Bid-Ask Spread}

Consider again the context of Section 3.2 (with patient sellers arriving faster than impatient buyers). The market is a Markov system with transition matrix $P$ as in equation (23). To calculate the distribution of the bid-ask spread, one needs to know the stationary probability that the system is in state $m$. Denote this by $x_{m}$. Define

$$
a=\frac{\omega}{1-\omega}, \quad \alpha=\frac{1-\omega}{\omega}, \quad \beta=\frac{1}{2 \omega-1} .
$$

Consider the row vector $X$ with entries $x_{m}$. From the theory of Markov matrices, one knows that $X P=X$. Solving for $X$, one gets $(1-\omega) x_{m+1}=\omega x_{m}$ for all $m$, hence $x_{m}=C\left(\frac{\omega}{1-\omega}\right)^{m}=$ $C a^{m}$. The components $x_{m}$ must sum to one, so $C=\frac{a-1}{a^{M+1}-1}$. Then

$$
x_{m}=\frac{a^{m+1}-a^{m}}{a^{M+1}-1} \approx \alpha^{M-m}-\alpha^{M-m+1} .
$$

To calculate the spread $a_{m}-B$, use the formula $f_{m-1}-f_{m}=a^{M-m} \varepsilon(\beta+u)-\varepsilon \beta$. From this point forward assume that there is no randomization in state $M$ (so $s=0$ and $u=\frac{1}{1-\omega}$ ). Then one gets the formula $f_{m-1}-f_{m}=\left(a^{M-m+1}-1\right) \varepsilon \beta$, which implies

$$
\begin{aligned}
a_{M}-B & =\varepsilon \beta(a-1), \\
a_{M-1}-B & =\varepsilon \beta\left((a-1)+\left(a^{2}-1\right)\right), \\
a_{M-2}-B & =\varepsilon \beta\left((a-1)+\left(a^{2}-1\right)+\left(a^{3}-1\right)\right),
\end{aligned}
$$

Notice that these spreads appear with stationary probabilities approximately equal to $1-$ $\alpha, \alpha-\alpha^{2}, \alpha^{2}-\alpha^{3}, \ldots$, respectively. It would be interesting to compare this distribution with the empirically observed one (especially at the tails of the distribution).

I give an example of calculating the first moment of the distribution, which may be of 
empirical interest. The exact formula for the bid-ask spread $\bar{S}$ is

$$
\bar{S}=\frac{\varepsilon \beta}{(a-1)\left(a^{M+1}-1\right)}\left((M+1) a^{M+3}-(M+3) a^{M+2}+(M+3) a-(M+1)\right) .
$$

This can be very well approximated by

$$
\bar{S} \approx \varepsilon \beta a M
$$

Now I give an asymptotic formula for $M$ when $\varepsilon$ is small. Recall from (14) that $M$ is obtained by solving $A-B+\varepsilon \beta M=\frac{\varepsilon \beta a^{M+1}}{a-1}\left(1-\alpha^{M}\right)$. When $\varepsilon$ is small, $M$ is large, so $a^{M}$ is much larger than $M$. Then one can drop $M$ from the above formula, and also approximate $1-\alpha^{M}$ with zero. One then obtains

$$
M \approx \frac{1}{\log a} \log \frac{1}{\varepsilon}
$$

So the average spread $\bar{S} \approx \beta \frac{a}{\log a} \varepsilon \log \frac{1}{\varepsilon}$, which implies that asymptotically

$$
\bar{S} \sim \varepsilon \log \frac{1}{\varepsilon}
$$

Compare this with Farmer et al. (2003), where $\bar{S} \sim \varepsilon^{3 / 4}$. (Notice that their $\varepsilon$, which they call "granularity parameter" is the same - up to a constant - as our $\varepsilon$, if one assumes that the patience $r$ of agents trading in different stocks is the same.)

\section{Conclusions}

This paper presents a tractable model of the dynamic of the limit order book. The shape of the limit order book and its evolution in time are characterized, in several cases in closed-form. The traders' optimal choices between submitting a limit order and a market order are also derived.

Having a good model of the limit order book should in principle generate many sharp predictions about the shape of the limit order book, and the evolution of buy and sell prices or of the bid-ask spread. I show that buy and sell orders can cluster away from the bid-ask spread, thus generating a hump-shaped limit-order book. Also, I explain why following a market buy order the both the bid and the ask increase, although the bid does not increase as 
much as the ask. An interesting empirical question that this model can address is to determine the behavior of asset returns, especially at high frequencies.

Some of the features of the model point to future avenues of research. One important direction is to study the interaction of agents in this model with a market maker. (An agent may decide to have a permanent presence in the market, and take advantage of the liquidity traders in the model - what are then the limits of arbitrage?) Another direction is to investigate how the range of prices $[B, A]$ arises endogenously, perhaps as the result of the interaction of traders with different valuations. This would amount to introducing information in the model, clearly a desirable feature.

Since in this paper trades are usually for one unit, one may wonder how to incorporate multi-unit trades. This is addressed in the paper, but agents do not have a choice over how many units to trade. A model where agents are strategic about this choice would surely be more complicated, but the payoff is that then one could define a meaningful notion of trading volume and analyze it within the model.

\section{A Proofs of Results}

Here is a proof of Proposition 2.

Proof. One needs to show that for each pair $A>B$ and each $\varepsilon>0$ there exists a unique maximal chain. First, let us solve in general the difference equation $2 f_{m}+\varepsilon=f_{m-1}+f_{m+1}$, with initial condition $f_{0}=A$. This is a quadratic function

$$
f_{m}=A-b m+\frac{\varepsilon}{2} m^{2}
$$

with $b$ some arbitrary real constant that has to be determined from the terminal condition, $f_{M}=B$. Now solve $f_{M}=B$, i.e.,

$$
A-M u \varepsilon-M(M-1) \frac{\varepsilon}{2}=B
$$

The positive solution is (if $R=\frac{2(A-B)}{\varepsilon}$ )

$$
M_{u}=\frac{1}{2}-u+\sqrt{\left(\frac{1}{2}-u\right)^{2}+R} .
$$


Notice that $M_{0}=\frac{1}{2}+\sqrt{\frac{1}{4}+R}$ and $M_{1}=-\frac{1}{2}+\sqrt{\frac{1}{4}+R}$, so $M_{0}-M_{1}=1$. Therefore in the interval $\left[M_{1}, M_{0}\right)$ there is a unique integer $M$ which corresponds to a number $u \in(0,1]$. This yields $M$ and $u$, and now $b=\varepsilon\left(M-\frac{1}{2}+u\right)$.

I now prove Theorem 9. Assume that there is a rigid competitive stationary Markov equilibrium of the game. Then one proceeds to study necessary conditions for this equilibrium to exist.

Definition 6. A state is called

a) full if agents leave the state only if some new agent arrives;

b) partial if an existing agent voluntarily exits after a positive expected waiting time;

c) fleeting if agents only stay in this state an infinitesimal time.

A state is called regular if it is either full or partial, so that agents will wait in it at least a positive amount of time.

I now investigate the state region $\Omega$, which is the collection of all regular states.

Proposition 14. There exist a finite number of states in $\Omega$ (with probability one).

Proof. Since agents lose utility proportionally to expected waiting time, there is a maximum expected time they will wait. Because of Poisson arrivals of the other agents, with probability one only a finite number of agents will arrive during this period (which is fixed in expectation). Now one has only to put this together with the fact that strategies of players in this game have all a uniformly bounded number of jumps (see Appendix B).

Definition 7. In any state $S$ denote by $f_{S}$ the value function of the sellers and by $g_{S}$ the value function of the buyers.

I already assumed the equilibrium is rigid, which means that at the boundary $\gamma$ some agents behave using mixed strategies, in such a way that buyers and sellers have the same value function. Then one has the following result:

Proposition 15. In a regular state $f_{S} \geq g_{S}$. In a partial or fleeting state $f_{S}=g_{S}$. 
Proof. To prove the first statement suppose in a regular state $f_{S}<g_{S}$. Then there exists $u>0$ such that $f_{S}<g_{S}-u$. So one of the sellers is better off offering at $g_{S}-u$, which one of the buyers is better off accepting. For the second result, one knows that by definition in all partial states $S$ one has $f_{S}=g_{S}$. Now each fleeting state $S$ corresponds along the main diagonal to a partial state. Now use Proposition 19 to show that $f_{S}=g_{S}$ as well (it is the only possible equilibrium).

Now I prove an important result, which allows one to determine the shape of the state region. It says that when a new seller arrives, every seller is worse off. Also, every buyer is better off, but by less than every seller is worse off.

Proposition 16. Suppose the system is in a regular state $S$ and a new seller arrives, so the system moves to regular state $S^{\prime}$. Then $f_{S^{\prime}}<f_{S}$ and $g_{S^{\prime}}>g_{S}$. Moreover,

$$
f_{S}-f_{S^{\prime}} \geq g_{S}-g_{S^{\prime}}
$$

Proof. The difficult inequality is the last one. If $S^{\prime}$ is a partial state, then $f_{S^{\prime}}=g_{S^{\prime}}$ and the proof is done. So suppose $S^{\prime}$ is a full state. The statement must be hardest to prove for one buyer, so I show how to prove it in that case. Even in the most optimistic scenario

$$
g_{S^{\prime}} \geq B+\frac{\varepsilon}{2}
$$

Also, one can assume that no extra buyers are arriving (one can prove the statement for each number of buyers). Then

$$
g_{S} \leq B+\varepsilon,
$$

because the buyer just has to wait for an impatient seller. One gets

$$
g_{S}-g_{S^{\prime}} \leq \frac{\varepsilon}{2}
$$

Now for $f$ : All the top agents in $S^{\prime}$ have to wait at least until an impatient buyer or patient (that becomes impatient) arrives into $S$. So one has

$$
f_{S}-f_{S^{\prime}} \geq \frac{\varepsilon}{2},
$$


from which one deduces that $f_{S}-f_{S^{\prime}} \geq g_{S}-g_{S^{\prime}}$.

Corollary 17. If a state $S=(m, n)$ is regular and $m>0, n>0$, then $(m-1, n)$ and $(m, n-1)$ are also regular.

Proof. Follows from the previous result.

Let $\Omega$ be a region in the positive quadrant of the plane which is symmetric with respect to the main diagonal. Suppose $\Omega$ has the property that if $(m, n)$ are in $\Omega$, then so are $(m-1, n)$ and $(m, n-1)$. Let $R$ be the largest integer such that $(0, R)$ is in $\Omega$. Define a function $\phi:\{0,1, \ldots, R\} \rightarrow \mathbb{N}$ in the following way: if $k=0,1, \ldots, R$ look at the intersection of the diagonal from the point $(0, R-k)$ with the boundary of $\Omega$. This is a point of the form $(0+j, R-k+j)$ for some $j \in \mathbb{N}$. Then define $\phi(k)=j$. Call $\phi$ the associated function, or the shape function of $\Omega$. The following result is not hard to prove.

Lemma 18. Let $\Omega$ be a symmetric integer region in the positive quadrant, with the property that if $(m, n)$ are in $\Omega$, then so are $(m-1, n)$ and $(m, n-1)$. Then the shape function $\phi$ is always increasing by either 0 or 1 . Conversely, any such function leads to a region $\Omega$ with the properties mentioned above.

I now analyze more carefully what happens in each state $(m, n)$. For this, take the onesided story, and assume there are $m$ sellers which compete for a bid of $h$. One can then prove the following important result, which is the game of attrition with Poisson arrivals.

Proposition 19. Suppose $m$ sellers lose utility in a way proportional to expected waiting time and coefficient $r$. At random time $T$ which represents the first arrival in a Poisson process with intensity $\lambda$, an event happens and the game ends (this event can be the arrival of a new agent). Then, if all sellers wait until $T$, assume that each gets a payoff of $f^{\infty}$. Also, at each time there exists a buyer who posts a bid for $h$. Assume that if a seller accepts $h$ until $T$, he gets $h$ and all other sellers get $f^{-}$. Denote by $f^{0}=f^{\infty}-r / \lambda$. Then one has the following list of possible sub-game perfect equilibria:

- If $h>\max \left\{f^{0}, f^{-}\right\}$, then every seller immediately accepts $h$ (and only one randomly gets it).

- If $h<\min \left\{f^{0}, f^{-}\right\}$, then no seller accepts $h$, and everybody waits until $T$. 
- If $h \in\left[f^{-}, f^{0}\right]$ and $f^{-} \leq f^{0}$, there are two SPE: either everybody waits until $T$ (this is the Pareto optimal equilibrium); or, if they believe the others will try to get $h$, they are all better off by doing the same: so each seller places a market order for $h$.

- $h \in\left[f^{0}, f^{-}\right]$and $f^{0} \leq f^{-}$, then this is a typical game of attrition. It has two equilibria: one, where some agent always accepts $h$, and the other never accept $h$; and the other where all agents accept $h$ according to some Poisson process with intensity $\mu$ ( $\mu$ is such that each agent is indifferent between accepting $h$ now and waiting for the other $m-1$ sellers to do that).

Now, the previous result does not assume anything about sellers placing limit orders. The next result is a simple extension of this game of attrition, where sellers are allowed to place limit orders. Clearly, the ask price is important now, because that might influence the payoff $f^{\infty}$ at $T$. I show that one gets one more equilibrium.

Corollary 20. In the setup of the previous proposition, suppose $\lambda=\lambda_{1}+\lambda_{2}$, where $\lambda_{1}$ is the Poisson intensity of the arrival of an impatient buyer (event 1), and $\lambda_{2}$ corresponds to any other event that does not depend on the value of the ask price (event 2). Now suppose that at $T$, if event 1 happens, the bottom seller (at the ask) gets the ask price, while all the other get $f_{1}^{\infty}$. If event 2 happens, assume that all sellers get $f_{2}^{\infty}$. Then, besides the equilibria above there is one more equilibrium, where the top sellers wait and the bottom seller randomly accepts $h$ with Poisson( $\mu)$, where $\mu$ is in such a way that everybody's value function is $h$.

This last equilibrium indicates exactly how agents behave in partial rigid states.

Proposition 21. Consider the state space $\Omega$ of a competitive stationary Markov equilibrium. Then, along each line parallel to the main diagonal there are initial full states, then at most one partial state, and then fleeting states. If the equilibrium is also rigid, then there is exactly one partial state.

Proof. Follows from Proposition 16 and Corollary 20.

Now I prove Theorem 11.

Proof. I show that $\triangle f=1$. Start with equation $4 f_{m, n}+\varepsilon=f_{m-1, n}+f_{m+1, n}+f_{m, n-1}+f_{m, n+1}$, 
and divide throughout by $\varepsilon=\delta^{2}$. Then one gets

$$
\frac{f_{m-1, n}-2 f_{m, n}+f_{m+1, n}}{\delta^{2}}+\frac{f_{m, n-1}-2 f_{m, n}+f_{m, n+1}}{\delta^{2}}=1 .
$$

But this is the finite difference approximation of the PDE

$$
\left(\frac{\partial^{2} f}{\partial x^{2}}+\frac{\partial^{2} f}{\partial y^{2}}\right)(m \delta, n \delta)=1
$$

which is exactly $\triangle f(x, y)=1$.

Now equation $3 f_{m, 0}+\varepsilon=f_{m-1,0}+f_{m+1,0}+f_{m, 1}$ becomes after division by $\delta$ :

$$
\frac{f_{m-1,0}-2 f_{m, 0}+f_{m+1,0}}{\delta^{2}} \cdot \delta+\frac{f_{m, 1}-f_{m, 0}}{\delta}=\delta
$$

After passing to the limit when $\delta$ goes to zero, one gets

$$
\frac{\partial f}{\partial y}(x, 0)=0
$$

If one picks a point on $\gamma$ of type 1 , one has $\left(4+s_{m, n}\right) f_{m, n}+\varepsilon=2 f_{m-1, n}+2 f_{m, n-1}+$ $s_{m, n} f_{m-1, n-1}$, which after division by $\delta$ becomes

$$
2 \frac{f_{m, n}-f_{m-1, n}}{\delta}+2 \frac{f_{m, n}-f_{m-1, n}}{\delta}+s_{m, n} \frac{f_{m, n}-f_{m-1, n-1}}{\delta}=-\delta .
$$

After passing to the limit when $\delta$ goes to zero, one gets

$$
\frac{\partial f}{\partial x}(x, y)+\frac{\partial f}{\partial y}(x, y)=0
$$

For a point on $\gamma$ of type 2 , one has $\left(4+s_{m, n}\right) f_{m, n}+\varepsilon=f_{m-1, n}+2 f_{m, n-1}+f_{m, n+1}+$ $s_{m, n} f_{m-1, n-1}$, which becomes

$\frac{f_{m, n}-f_{m-1, n}}{\delta}+\frac{f_{m, n}-f_{m-1, n}}{\delta}+\frac{f_{m, n-1}-2 f_{m, n}+f_{m, n+1}}{\delta^{2}} \cdot \delta+s_{m, n} \frac{f_{m, n}-f_{m-1, n-1}}{\delta}=-\delta$.

In the limit one gets the same condition $\frac{\partial f}{\partial x}(x, y)+\frac{\partial f}{\partial y}(x, y)=0$.

Finally, the condition $f=g$ on $\gamma$ is obvious. 


\section{B Continuous Time Game Theory}

In this paper the strategic interaction of agents is modeled as a multi-stage game with observed actions, ${ }^{20}$ but in continuous time. The framework I use borrows heavily from the theory of repeated games in continuous time, as developed by Bergin and MacLeod (1993). Since there are also stochastic moves by Nature and entry decisions by new players, I extend their framework by using an idea from Simon and Stinchcombe (1989).

Since this is a trading game, one may wonder why I consider simultaneous moves (organized in the "stage game" at each time $t$ ), and not have only successive moves by players. (Indeed, on organized exchanges there is a strict priority of both market and limit orders.) The reason is that it is technically easier to describe a game in continuous time where agents move simultaneously; otherwise, the submission order would have to be decided by a randomization procedure. Also, one may want successive actions to happen "instantly", which would force one to consider them all at the same $t$.

There is however a cost to be paid: extra care has to be taken about market orders. To see why, suppose at some time $t$ a trader submits a market buy order, and simultaneously the bid and ask prices are determined by the limit orders placed by the other players. Then in equilibrium all sellers would compete to place a limit order at the top price $A$. This contradicts the intuition that the market order should be executed at the existing ask - which was set right before $t$-and not at the new ask set at $t$. One way to get around this problem is to demand that market orders have to be cleared at the bid or ask prices set right before $t$ (of course, one will have to define precisely what "right before" means in continuous time). The other problem with market orders is that they reduce the number of players in the game. Since I want this event to happen at a definite time, I require that when a market order is submitted at $t$, the game between the remaining players takes place also at $t$. One way to get around this problem is by introducing "layered times" (see below), i.e., by allowing multiple games to played at a discrete set of times in $[0, \infty)$. This is reminiscent of auction theory, and the notion of "stopping the clock."

Now I discuss multi-stage games with observed actions in continuous time. I start with the case of pure strategies, and later in the section I deal with mixed strategies. Game theory in continuous time is not a straightforward extension of game theory in discrete time. There

\footnotetext{
${ }^{20}$ See Fudenberg and Tirole (1991), chapter 4.
} 
are a few conceptual difficulties, as pointed out by Simon and Stinchcombe (1989), or Bergin and MacLeod (1993). To understand why, suppose one tries to replicate a typical punishment strategy from discrete time repeated games:

Continue to cooperate if the other player has not defected yet; if the other player defected at any point in the past, immediately defect and continue to defect forever.

The difficulty to make this strategy precise is two-fold. First, in continuous time there is no first time after $t$, which makes it difficult to "continue" a certain course. One way to get around this problem is to allow strategies to have inertia. But this creates a second problem, since the other players can take advantage of inertia. ${ }^{21}$ One way to allow players to react "immediately" is to enlarge the concept of strategy to include sequences of faster and faster responses. The mathematical concept that allows us to do that is completion with respect to a metric (see below). ${ }^{22}$

A third problem that arises in continuous time is that there is no first time before $t$. This issue is important when one needs a description of the game right before $t$. For example, in a trading game, suppose a trader submits a market buy order at $t$. This market order is supposed to be very fast, and not give time for the existing traders to change their limit orders - otherwise, all sellers would try to change their limit order at the highest possible level $A$. One can then model this by allowing the market order to be placed "immediately before" $t$, and the game at $t$ will be played with one less player, namely the trader whose limit order was cleared. Since there is no first time before $t$, it is not obvious at which price the market order is to be executed. The solution of this problem is to allow only strategies that "behave well" immediately before any time $t$. The technical concept, borrowed from Simon and Stinchcombe (1989), is of a strategy with a uniformly bounded number of jumps (to be defined below).

In order to define a game, one must define the spaces of actions, outcomes, and strategies. The definitions follow closely those of Bergin and MacLeod (1993). I extend their framework in several directions: (i) there is a well-defined description of the game right before any time $t$; (ii) I allow for entry decisions of new agents; and (iii) I account for the possibility of having more than one game played at the same time. I start with an infinitely countable set of players

\footnotetext{
${ }^{21}$ One could force the players to all have the same inertia, but then this would be equivalent to forcing the game to take place in discrete time.

${ }^{22}$ Another way to define immediacy is by using infinitesimal numbers, which is the mathematical field of non-standard analysis.
} 
I. Since they arrive according to independent Poisson processes, with probability one at each point in time there are only finitely many traders. I want to include the case where at some times $t$ the game is played more than once. I do this by taking the product of the time interval $[0, \infty)$ with the set of natural numbers $\mathbb{N}$, to indicate how many time a game has been played at some time $t$. Define

$$
\mathcal{T}=[0, \infty) \times \mathbb{N}
$$

the set of times at which players can move, counted with multiplicity. Notice that if $\leq$ is the lexicographic order, $(\mathcal{T}, \leq)$ is a totally ordered space. Denote the element $(0,0) \in \mathcal{T}$ also by 0 . Define intervals in $\mathcal{T}$ in the usual way: for example, if $T=(t, n) \in \mathcal{T}$ define $[0, T)=\left\{T^{\prime} \in \mathcal{T} \mid 0 \leq T^{\prime}<T\right\}$. When there is no danger of confusion, write $t$ instead of $(t, n)$. Also, define a measure on $[0, \infty)$ so that bounded measurable functions are integrable:

$$
\mu(\mathrm{d} t)=\mathrm{e}^{-a t} \mathrm{~d} t
$$

In general, I want the action space for player $i$ to be a compact complete metric space $\left(X_{i}, d_{i}\right)$. Typically, $X_{i}$ is a compact subset of $\mathbb{R}^{n}$ and $d_{i}$ is the inherited metric. In this paper, the action space for player $i \in I$ can be defined as a subset of $\mathbb{R}^{2}$ :

$$
X_{i}=([B, A] \times\{0,1\}) \cup\{\text { out }\},
$$

where out is some point in $\mathbb{R}^{2}$ which does not lie on $[B, A] \times\{0,1\}$. An action $\left(x_{i}, 1\right) \in X_{i}$ is interpreted as a limit order at $x_{i} \in[B, A]$. An action $\left(x_{i}, 0\right) \in X_{i}$ is interpreted as a market sell (buy) order, in which case $x_{i}$ is the current bid (ask) price, respectively. The action $x_{i}=$ out indicates that either (i) player $i$ has not entered the game yet; or (ii) player $i$ exited the game before. One could also allow agents to exit freely at time $t$. This will not happen in equilibrium if the utility from exiting is very small, so in order to simplify the description of the game I do not allow free exit. Define also projections on the first and second factor, $\pi_{1}: X_{i} \rightarrow[B, A] \cup\{$ out $\}$ and $\pi_{2}: X_{i} \rightarrow\{0,1\} \cup\{$ out $\}$, in the obvious way.

I now define outcomes of the game. Let $\mathcal{B}_{X_{i}}$ and $\mathcal{B}_{X}$ be the Borel sets of $X_{i}$ and $X=$ $\prod_{i \in I} X_{i}$, respectively; and let $\mathcal{B}$ be the Borel sets of $[0, \infty)$. A function $\nu:[0, \infty) \rightarrow \mathbb{N}$ is said to have finite support if $\nu$ is zero everywhere except on a finite set $M_{1}=\left\{t_{1}, \ldots, t_{K}\right\}$ (its support). One also associates the set $M=\left\{\left(t_{1}, n_{1}\right), \ldots,\left(t_{K}, n_{K}\right)\right\}$, where all $n_{k}=\nu\left(t_{k}\right)>0$. 
Vice versa, for any such set $M$ one can define a function $\nu_{M}:[0, \infty) \rightarrow \mathbb{N}$ with finite support by sending $t \in[0, \infty)$ to zero if $t \notin M_{1}$; and to $n_{k}$ if $t=t_{k} \in M_{1}$.

Definition 8. Let $X$ be a space with measure. A function $f: \mathcal{T} \rightarrow X$ is called layered if there exists a function $\nu:[0, \infty) \rightarrow \mathbb{N}$ with finite support such that $\forall t \in[0, \infty)$ and $\forall n, n^{\prime}>\nu(t)$ one has $f(t, n)=f\left(t, n^{\prime}\right)$. If $f: \mathcal{T} \rightarrow X$ is layered, associate a function $f^{\nu}:[0, \infty) \rightarrow X$ by $f^{\nu}(t)=f(t, \nu(t))$. I say that $f$ is a layered measurable function if $f^{\nu}$ is measurable. An outcome for player $i$ is a layered Borel measurable function $h_{i}: \mathcal{T} \rightarrow X_{i}$.

So an outcome is like a regular measurable function $h_{i}:[0, \infty) \rightarrow X_{i}$, except that at a finite set $\left\{t_{1}, \ldots, t_{K}\right\}$ (the support of $\nu$ ) it can take several values, up to the integer number $\nu\left(t_{k}\right)$. This corresponds to the idea that at some times $t_{k}$ the game can be played more than once (in my case, if some agent places a market order).

I call the function $\nu$ the layer of $f$. Sometimes I also call the layer of $f$ the associated set $M=\left\{\left(t_{1}, n_{1}\right), \ldots,\left(t_{K}, n_{K}\right)\right\}$, with $n_{k}=\nu\left(t_{k}\right)$. Also, if $f_{1}$ and $f_{2}$ are two layered functions with layers $\nu_{1}$ and $\nu_{2}$, one can take the combined layer of $f_{1}$ and $f_{2}$ to be $\nu=\max \left\{\nu_{1}, \nu_{2}\right\}$. This is useful for situations where one has to compare $f_{1}$ and $f_{2}$. Consider a layer $\nu$. Then I define: $\mathcal{T}^{\nu}$, the set of layered times associated with $\nu ; H_{i}$, the space of outcomes for player $i$; and $H_{i}^{\nu}$, the space of outcomes associated with $\nu$ :

$$
\begin{gathered}
\mathcal{T}^{\nu}=\{(t, n) \in \mathcal{T} \mid n \leq \nu(t)\}, \\
H_{i}=\left\{h_{i}: \mathcal{T} \rightarrow X_{i} \mid h_{i} \text { layered measurable }\right\}, \\
H_{i}^{\nu}=\left\{h_{i}: \mathcal{T} \rightarrow X_{i} \mid h_{i} \text { layered measurable with layer } \nu\right\} .
\end{gathered}
$$

This is a metric space with the metric $D_{i}: H_{i}^{\nu} \times H_{i}^{\nu} \rightarrow \mathbb{R}_{+}$given by

$$
D_{i}\left(h_{i}, h_{i}^{\prime}\right)=\int_{[0, \infty)} d_{i}\left(h_{i}^{\nu}(t), h_{i}^{\prime \nu}(t)\right) \mu(\mathrm{d} t)+\sum_{k=1}^{K} \sum_{n=0}^{\nu\left(t_{k}\right)} d_{i}\left(h_{i}\left(t_{k}, n\right), h_{i}^{\prime}\left(t_{k}, n\right)\right) .
$$

Rewrite this as

$$
D_{i}\left(h_{i}, h_{i}^{\prime}\right)=\int_{\mathcal{T}^{\nu}} d_{i}\left(h_{i}(T), h_{i}^{\prime}(T)\right) \mu^{\nu}(\mathrm{d} T)
$$

Since the space of measurable functions $f_{i}:[0, \infty) \rightarrow X_{i}$ is compact and complete, so is $H_{i}^{\nu}$. Now, if $\nu \leq \nu^{\prime}$, there is an inclusion $H_{i}^{\nu} \rightarrow H_{i}^{\nu^{\prime}}$. Also, one knows that for every two layers $\nu_{1}$ and $\nu_{2}$ one can take their maximum $\nu=\max \left\{\nu_{1}, \nu_{2}\right\}$, which satisfies $\nu_{1}, \nu_{2} \leq \nu$. This means 
that one can regard $H_{i}$ as the limit of $H_{i}^{\nu}$ when $\nu$ becomes larger and larger. Because of this, $H_{i}$ is a metric space, but it might not be either complete or compact.

I now define the space $H$ of outcomes of the game. For this, let $H^{\nu}=\prod_{i \in I} H_{i}^{\nu}$ the product space with the metric $D=\prod_{i \in I} \frac{1}{2^{i}} D_{i}$. It is a standard exercise in measure theory to see that $H^{\nu}$ is compact and complete. As before, if $\nu \leq \nu^{\prime}$, there is an inclusion $H^{\nu} \rightarrow H^{\nu^{\prime}}$. I then define $H$ as the union of all $H^{\nu}$ for all layers $\nu$. This is still a metric space, but it might not be complete or compact. To justify this definition, consider an outcome $h \in H$. Since $h$ belongs to a union of $H^{\nu}$ over all layers $\nu$, there must exist a particular $\nu$ so that $h \in H^{\nu}$ (in which case, I say that $\nu$ is the layer of $h$ ). This corresponds to the fact that all agents are in the same game, played at the times described by the layer $\nu$.

Also, if $Z \subset \mathcal{T}^{\nu}$ is layered measurable, and $h_{i}, h_{i}^{\prime} \in H_{i}^{\nu}$, define a metric relative to $Z$ by $D_{i}\left(h_{i}, h_{i}^{\prime}, Z\right)=\int_{Z} d_{i}\left(h_{i}(T), h_{i}^{\prime}(T)\right) \mu^{\nu}(\mathrm{d} T)$. Define also a metric $D$ on $H$ relative to $Z$ in the same way it was done for the product metric above.

Now I define strategies. In discrete time, pure strategies map histories to actions, while mixed strategies map histories to probability densities over actions. For technical reasons it is easier to think of a history as an outcome of the game together with a time $t$ at which history is taken. This way, one can define a strategy as a map from $\{$ outcomes $\times$ times $\}$ to $\{$ actions $\}$. Formally, a strategy for agent $i$ is a map

$$
s_{i}: H \times \mathcal{T} \rightarrow X_{i}
$$

which satisfies the following axioms

A1. The function $s_{i}$ is layered measurable on $H \times \mathcal{T}$.

A2. For all $h, h^{\prime} \in H$ and $T \in \mathcal{T}$ such that $D\left(h, h^{\prime},[0, T)\right)=0$, one has $s_{i}(h, T)=s_{i}\left(h^{\prime}, T\right)$.

The second axiom ensures that future does not affect current decisions. Rewrite

$$
h \sim_{T} h^{\prime} \Longleftrightarrow D\left(h, h^{\prime},[0, T)\right)=0
$$

As it was discussed above, these two axioms alone do not ensure that strategies uniquely determine outcomes. For that, one needs some inertia condition. If $t \in[0, \infty)$ and $\nu$ is a layer, denote by $t^{\nu}=(t, \nu(t))$, and $t=(t, 0)$. 
A3. The function $s_{i}$ displays inertia, i.e., for any $h \in H^{\nu}$ and any $t \in[0, \infty)$, there exists $\varepsilon>0$ and $x_{i} \in X_{i}$ such that

$$
D_{i}\left(s_{i}\left(h^{\prime}\right), x_{i},\left[t^{\nu}, t+\varepsilon\right)\right)=0
$$

for every $h^{\prime} \in H^{\nu}$ such that $h \sim_{t^{\nu}} h^{\prime}$.

Denote by $S_{i}$ the set of functions $s_{i}$ on $H \times \mathcal{T}$ which satisfy A1, A2, A3. Denote by $S=\prod_{i \in I} S_{i}$. The next theorem shows that a strategy profile $s=\left(s_{i}\right)_{i}$, i.e., a set of strategies $s_{i}$ for for each player $i \in I$, uniquely determine an outcome on every subgame. More precisely one has the following result:

Proposition 22. Let $s \in S$. Then for every $h \in H$ and $T \in \mathcal{T}$, there exists a unique (continuation) outcome $\tilde{h} \in H$ so that $h \sim_{T} \tilde{h}$ and $D(s(\tilde{h}), \tilde{h},[T, \infty))=0$.

Proof. The proof is the same as in Bergin and MacLeod, but one has to make sure that one works in $H^{\nu}$ for some layer $\nu$.

Given $(h, T) \in H \times \mathcal{T}$ and $s \in S$, denote by $\sigma(s, h, t)$ the outcome which agrees with $h$ on $[0, T)$ and is determined by the strategy $s$ on $[T, \infty)$. Let $s_{i}, s_{i}^{\prime} \in S_{i}$. I now define a metric on $S_{i}$ :

$$
\rho_{i}\left(s_{i}, s_{i}^{\prime}\right)=\sup _{H \times \mathcal{T} \times S_{-i}} D\left(\sigma\left(\left(s_{i}, s_{-i}\right), h, T\right), \sigma\left(\left(s_{i}^{\prime}, s_{-i}\right), h, T\right)\right) .
$$

One also has to introduce an axiom which ensures that for each $t$ the outcome of the game right before $t$ is well defined. One way of doing this is to restrict to strategies $s_{i}$ that lead to locally constant outcomes with a uniformly bounded number of jumps.

A4. For the strategy $s_{i}$ there exists $M$ (depending only on $s_{i}$ ) such that for any strategies $s_{-i}$ of the other players, the outcome $\sigma_{i}\left(\left(s_{i}, s_{-i}\right), h, t\right)$ for player $i$ is locally constant and has at most $M$ jumps.

Redefine $S_{i}$ to include on the strategies that satisfy A4. Now recall that at each $t \in[0, \infty)$ the strategies have inertia for some $\varepsilon$ (depending on $t$ ). I want inertia to be infinitesimal, because I want to allow for immediate responses. This can be done by completing the space of strategies: Denote by $S_{i}^{*}$ the completion of $S_{i}$ with respect to the metric $\rho_{i}$, and by $S^{*}=\prod_{i \in I} S_{i}^{*}$. Completion is done so that the upper bound for the number of jumps is the same for all. 
More precisely, a point in $S_{i}^{*}$ corresponds to a Cauchy sequence $\left(s_{i}^{n}\right)_{n}$ of strategies in $S_{i}$, and one demands that there exists $M$ so that for each $n, s_{i}^{n}$ jumps at most $M$ times, regardless of the other players' strategies. The following result shows that to each strategy in $S^{*}$ one can associate a unique outcome in $H$.

Proposition 23. For every $s \in S^{*}$ and every $(h, T) \in H \times \mathcal{T}$, there exists a unique $h^{*}$ such that $\sigma\left(s^{n}, h, T\right) \rightarrow h^{*}$ for any Cauchy sequence $\left(s^{n}\right)_{n}$ in $S$ converging to $s$.

Proof. If $(h, T) \in H \times \mathcal{T}$, there exists a layer $\nu$ so that $h \in H^{\nu}$ and $T \in \mathcal{T}^{\nu}$. The result then follows easily since $H^{\nu}$ is compact and complete.

I have just showed that for $s \in S^{*}$ one can associate a unique outcome of the (whole) game, which I denote by $\sigma^{*}(s)$. Because completion is done using the same upper bound for the number of jumps, the following result is straightforward. The result allows one to talk about the outcome of a game right before some time $t$.

Proposition 24. The outcome $\sigma^{*}(s)$ associated to a strategy $s \in S^{*}$ is left continuous.

I am almost done in defining the game. The only thing that is left is to describe the payoff for some strategy $s \in S^{*}$ in a subgame defined by a history $(h, T) \in H \times \mathcal{T}$. Since strategies uniquely define outcomes in every subgame, as long as there exists some payoff $u_{i}\left(\sigma^{*}(s, h, T)\right)$ for each agent $i$. Define now the equilibrium concept:

Definition 9. A strategy profile $s \in S^{*}$ is an $\varepsilon$-Nash equilibrium $(\varepsilon-N E)$ if for any $h \in H$

$$
u_{i}\left(\sigma^{*}(s, h, 0)\right) \geq u_{i}\left(\sigma^{*}\left(\left(s_{i}^{\prime}, s_{-i}\right), h, 0\right)\right), \quad \forall i \in I, \quad \forall x_{i}^{\prime} \in S_{i}^{*} .
$$

A strategy profile $s \in S^{*}$ is an $\varepsilon$-subgame perfect Nash equilibrium $(\varepsilon-S P E)$ if for any $(h, T) \in$ $H \times \mathcal{T}$

$$
u_{i}\left(\sigma^{*}(s, h, T)\right) \geq u_{i}\left(\sigma^{*}\left(\left(s_{i}^{\prime}, s_{-i}\right), h, T\right)\right), \quad \forall i \in I, \quad \forall x_{i}^{\prime} \in S_{i}^{*}
$$

For $\varepsilon=0$ in the above inequalities one obtains the concepts of Nash equilibrium (NE) and subgame perfect Nash equilibrium (SPE). One has the following important result.

Proposition 25. A strategy profile $s \in S^{*}$ is a subgame perfect equilibrium if and only if for any Cauchy sequence $\left(s^{n}\right)_{n}$ converging to $s$, there is a sequence $\varepsilon^{n} \rightarrow 0$ such that $s^{n}$ is an $\varepsilon^{n}$-subgame perfect equilibrium. 
I now discuss mixed strategies, or rather, as they will be called, "pure-mixed." For simplicity of discussion I omit the presence of layers, so one takes $\mathcal{T}=[0, \infty)$. Consistent with this philosophy of locally constant outcomes and inertia strategies, I want to have mixed strategies randomly switch over a small interval. More formally, let $X_{i}$ be the space of actions for player $i$, and $[0, \infty]$ the metric space with metric $d(x, y)=\left|\mathrm{e}^{-x}-\mathrm{e}^{-y}\right|$. Define a mixed strategy to be a layered measurable function

$$
s_{i}: H \times \mathcal{T} \rightarrow X_{i} \times X_{i} \times[0, \infty],
$$

where the first component of $s_{i}$ is the initial action in $X_{i}$; the second component is the action to which $s_{i}$ will switch in the interval of time right after $t$; the third component is the Poisson intensity of switching. I call this type of strategy pure-mixed, because randomness only comes from the time of switching, while the actions before or after switching are deterministically chosen. One could in principle also allow for randomization over these actions, in which case one has to replace $X_{i}$ by $\Phi\left(X_{i}\right)$, the set of probability densities over $X_{i}$, i.e., the set of non-negative integrable functions on $X_{i}$ with total integral equal to one. But then the analysis would become much more complicated, and these strategies are not really necessary for the games considered in this paper.

I say that the strategy $s_{i}$ has inertia in a similar way as before, but one adds the requirement that after switching the value to which player $i$ switched will be also held constant for a small period of time. Then one has to modify the description of outcomes, which are now stochastic processes and are built in a very similar way to Poisson processes. The space of strategies is also constructed by taking a completion, in the same way it was done for pure strategies.

To see how a pure-mixed strategy works, consider the case of Nature, which moves at each time $t$. The space of actions for Nature is the set $2^{I}$ of all subsets of $I$ (in principle I allow Nature to add or remove any players from the game). Nature plays the following strategy: if $(h, t) \in H \times \mathcal{T}$ is the history at $t$, the first component of $s_{N}(h, t)$ is the set $J=I_{t_{-}}$of players right before $t$; the third component is the sum $\lambda=\lambda_{P S}+\lambda_{P B}+\lambda_{I B}+\lambda_{I S}$ of the arrival intensities of all four types of traders (patient sellers, etc.); the second component is a density over $2^{I}$ given by $\phi(J \cup\{P B\})=\frac{\lambda_{P B}}{\lambda}, \phi(J \cup\{P S\})=\frac{\lambda_{P S}}{\lambda}, \phi(J \cup\{I B\})=\frac{\lambda_{I B}}{\lambda}$, $\phi(J \cup\{I S\})=\frac{\lambda_{I S}}{\lambda}$, and $\phi(Z)=0$ for any other set $Z \in 2^{I}$. 
I now briefly discuss the notions of equilibrium employed in this paper. The main one is that of a sub-game perfect equilibrium (SPE), which reflects the dynamic nature of the game. But, in order to formalize some other intuitions which are important for this game, I also consider several refinements. As discussed before, there can be many SPE of this game, for example the ones based on Nash threats (as in the theory of repeated games). However, if one focuses on "competitive" equilibria, i.e., equilibria where agents do not take into consideration what effect their action has on the other players' strategies, one sees that the the competitive equilibria are essentially unique (very close to each other).

In this context the notion of competitive equilibrium is very close to that of a Markov perfect equilibrium $^{23}$ (MPE), where the state variable is $(m, n)$, with $m$ the number of sellers and $n$ the number of buyers active in the game at a particular time. A Markov or equilibrium is stationary if the value function only depends on the state $(m, n)$ and not on time $t$.

Competitive equilibria are important in a trading game framework, because they give the main intuition for the equilibrium limit order book. One can think of a competitive equilibrium as a Bertrand-type competition among patient traders for the stream of market orders of the impatient traders. Since execution of limit orders is subject to the FIFO (First-In-First-Out) policy, it follows that traders have different times-to-execution for their orders, and that means that in order to compete (and get the same expected payoff), patient agents have to place limit orders at different levels in the book. I then say that an SPE is competitive if at each time $t$ all agents have the same value function (possibly depending on $t$ ). I want to stress the fact that this definition is quite ad-hoc and is only valid in my context, where the symmetry of the payoffs is broken by the FIFO policy.

\section{References}

[1] Admati, A. And P. Pfleiderer (1988), A theory of intraday patterns: Volume and price variability, Review of Financial Studies 1, 3-40.

[2] Aminud, Y. And H. Mendelson (1980), Market-making with inventory, Journal of Financial Economics 8, 31-53.

\footnotetext{
${ }^{23}$ Let $k_{t}$ be a variable which summarizes what one wants to know about a game at time $t$. Suppose $k_{t}$ evolves according to a Markov process with transition function $q\left(k_{t+\mathrm{d} t} \mid k_{t}, a_{t}\right)$, which gives the probability that the next period's state is $k_{t+\mathrm{d} t}$ conditional on its being $k_{t}$ at time $t$ and on playing the actions $a_{t}$. By definition, if two histories lead to the same $k_{t}$, a Markov strategy maps them to the same action. A Markov perfect equilibrium is a perfect equilibrium in Markov strategies. Notice that the concept of MPE depends on the particular state variable chosen. See Fudenberg and Tirole (1991, ch. 13).
} 
[3] Bergin, J. and B. MacLeod (1993), Continuous time repeated games, International Economic Review 34, 21-37.

[4] Biais, B., P. Hillion and C. Spatt (1995), An empirical analysis of the limit order book and the order flow in the Paris Bourse, Journal of Finance 50, 1655-1689.

[5] Bouchaud, J-P., M. Mezard And M. Potters (2002), Statistical properties of the stock order books: empirical results and models, Mathematics ArXiv, http: //xxx.lanl.gov, cond-mat/0203511.

[6] Black, F. (1971), Towards a fully automated exchange, first part, Financial Analysts Journal 27, 29-34.

[7] Chakravarty, S. and C. Holden (1995), An integrated model of market and limit orders, Journal of Financial Intermediation 4, 213-241.

[8] Cohen, K., S. Maier, R. Schwartz and D. Whitcomb (1981), Transaction costs, order placement strategy, and existence of the bid-ask spread, Journal of Political Economy 89, 287-305.

[9] Demsetz, H. (1968), The cost of transacting, Quarterly Journal of Economics 82, 33-53.

[10] Domowitz, I. And A. WAng (1994), Auctions as algorithms, Journal of Economic Dynamics and Control 18, 29-60.

[11] Duffie, D., N. Garleanu and L. Pedersen (2001), Valuation in dynamic bargaining markets, Stanford University preprint, September.

[12] Evans, L. (1998), Partial Differential Equations, Graduate Studies in Mathematics, Vol. 19, American Mathematical Society.

[13] Farmer, D., P. Patelli And I. Zovko (2003), The predictive power of zero intelligence in financial markets, preprint, September.

[14] Foucault, T. (1999), Order flow composition and trading costs in a dynamic limit order market, Journal of Financial Markets 2, 99-134.

[15] Foucault, T., O. Kadan and E. Kandel (2003), Limit order book as a market for liquidity, preprint, October.

[16] Fudenberg, D. And J. Tirole (1991), Game Theory, MIT Press.

[17] Gabaix, X., P. Gopikrishnan, V. Plerou and E. Stanley (2003), Theory of large fluctuations in stock market activity, MIT preprint, August.

[18] Garman, M. (1976), Market microstructure, Journal of Financial Economics 3, 257275 .

[19] Glosten, L. (1994), Is the electronic open limit order book inevitable?, Journal of Finance 49, 1127-1161.

[20] Glosten, L. and P. Milgrom (1985), Bid, ask and transaction prices in a specialist market with heterogeneously informed traders, Journal of Financial Economics 14, 71100. 
[21] Goettler, R., C. Parlour and U. Rajan (2003), Equilibrium in a dynamic limit order market, Carnegie Mellon University preprint, May.

[22] Grossman, S. And M. Miller (1988), Liquidity and market structure, Journal of Finance 43, 617-633.

[23] Handa, P. And R. Schwartz (1996), Limit order trading, Journal of Finance 51, $1835-1861$.

[24] Harris, L. (1998), Optimal dynamic order submission strategies in some stylized trading problems, Financial Markets, Institutions and Instruments, Vol. 7, No. 2.

[25] Harris, L. And J. Hasbrouck (1996), Market versus limit orders: the Superdot evidence on order submission strategy, Journal of and Quantitative Analysis, 31, 213-231.

[26] Hasbrouck, J. and G. Sofianos (1993), The trades of market makers: an empirical analysis of NYSE specialists, Journal of Finance, 68, 1565-1593.

[27] Hausman, J., A. Lo, and C. McKinlay (1992), An ordered Probit analysis of transaction stock prices, Journal of Financial Economics 31, 319-330.

[28] Hollifield, B., R. Miller, P. Sandas And J. Slive (2002), Liquidity supply and demand in limit order markets, CEPR Discussion Paper No. 3676, December.

[29] Huang, R. And H. Stoll (1997), The components of the bid-ask spread: A general approach, Review of Financial Studies 10, 995-1034.

[30] JAIN, P. (2002), Institutional design and liquidity on stock exchanges around the world, working paper, Indiana University at Bloomington.

[31] Kumar, P. And D. Seppi (1993), Limit orders and market orders with optimizing traders, Carnegie Mellon preprint.

[32] Kyle, Albert (1985), Continuous auctions and insider trading, Econometrica 53, 13151335 .

[33] Lo, A., C. McKinlay and J. Zhang (2001), Econometric models of limit order executions, Journal of Financial Economics 65, 31-71.

[34] O'Hara, M. (1995), Market Microstructure Theory, Blackwell.

[35] Parlour, C. (1998), Price dynamics in limit order markets, Review of Financial Studies 11, 789-816.

[36] Rock, K. (1996), The specialist's order book and price anomalies, working paper.

[37] Seppi, D. (1997), Liquidity provision with limit orders and a strategic specialist, Review of Financial Studies 10, 103-150.

[38] Simon, L. And M. Stinchcombe (1989), Extensive form games in continuous time: pure strategies, Econometrica 57, 1171-1214.

[39] Vayanos, D. And T. WAng (2003), Search and endogenous concentration of liquidity in asset markets, MIT preprint. 
[40] Wilson, R. (1986), Equilibria of bid-ask markets, in: Arrow and the Ascent of Economic Theory: Essays in Honor of Kenneth J. Arrow, G. Feiwel (ed.), Macmillan Press. 